\title{
Computational Prediction of the Molecular Mechanism of Statin Group of Drugs Against SARS-CoV-2 Pathogenesis
}

\section{Dipanjan Ghosh}

Department of Biotechnology and Dr. B. C. Guha Centre for Genetic Engineering and Biotechnology, University of Calcutta

\section{Debabrata Ghoshdastidar}

Guru Nanak Institute of Pharmaceutical Science and Technology

\section{Kamalesh Roy}

Institute of Genetic Engineering

Arnab Ghosh

National Institute of Biomedical Genomics

\section{Nilabja Sikdar}

Indian Statistical Institute

\section{Nidhan Biswas}

National Institute of Biomedical Genomics

\section{Gopal Chakrabarti}

Department of Biotechnology and Dr. B. C. Guha Centre for Genetic Engineering and Biotechnology, University of Calcutta Amlan Das ( $\triangle$ amlan.res1980@gmail.com)

National Institute of Biomedical Genomics

\section{Research Article}

Keywords: Statins, SARS-CoV-2, Insilico Docking, Molecular Dynamics

Posted Date: August 16th, 2021

DOI: https://doi.org/10.21203/rs.3.rs-783266/v1

License: (c) (1) This work is licensed under a Creative Commons Attribution 4.0 International License. Read Full License

Version of Record: A version of this preprint was published at Scientific Reports on April 14th, 2022. See the published version at https://doi.org/10.1038/s41598-022-09845-y. 


\section{Abstract}

\section{Background}

The COVID-19 pandemic had raised a severe health concern across the globe, as most countries are fighting with the second wave of SARS-CoV-2 infection. Although several vaccines had cleared the clinical trials and are being administered in different countries, the degree of protection varies due to the emergence of novel viral strains. Moreover, no antiviral drug against SARS-CoV-2 was reported to date. Recently published reports on statin therapy on COVID-19 patients indicated that statin therapy is associated with a better clinical outcome and a significant reduction in mortality risk.

\section{Methods}

Blind docking of the critical structural and functional proteins of SARS-CoV-2 like RNA-dependent RNA polymerase, Mprotease of 3-CL-Pro, Helicase, and the Spike proteins ( wild type and mutants) with the statin molecules were performed using the Schrodinger docking tool. Prevalent mutations in the spike protein were determined by analyzing the SARS-CoV-2 genome sequences deposited in GISAID, using the NextAlign aligner tool. Wild type and mutant spike proteins were modeled using the Swiss Model server and subjected to energy minimization via YASARA server. The percentage of the allowed region was determined by the Ramachandran plot. The MD simulation studies of free protein templates and protein-ligand complex were performed using NAMD (Nanoscale Molecular Dynamics) program; v 2.8.

Results

We observed that fluvastatin and pitavastatin showed fair binding affinities to RNA polymerase, 3-CL-Pro, and spike-double mutant with similar docking scores. But of all the target proteins, fluvastatin showed the strongest binding affinity to the helicase with the glide score, emodel score, and glide energy values of $-11.333,-66.511$ and $-58.72(\mathrm{kcal} / \mathrm{mol})$, respectively. Pitavastatin having similar chemical properties like fluvastatin (logP and pKa values) exhibited strong biding affinities to RdRp, 3-CL-Pro, and S-double mutant. Additionally, molecular dynamics simulation confirmed the formation of a stable drugprotein complex.

Conclusion

Thus our study shows that of all the statins, fluvastatin can bind to multiple target proteins of SARS-CoV-2, including the spike-mutant proteins. This property might contribute to the potent antiviral efficacy of this drug.

\section{Background}

The COVID-19 pandemic caused by SARS-CoV-2 (Severe acute respiratory syndrome coronavirus 2, a novel coronavirus strain) has imposed a severe threat to humanity. (SARS-CoV-2) also referred to as 2019 novel coronavirus (2019-nCoV) or human coronavirus 2019 (hCoV-19) (1) is a positive-sense single-stranded RNA virus responsible for the highly contagious severe acute respiratory syndrome (SARS) in humans (2). By the first week of May 2021, the number of COVID-19 confirmed cases had surpassed 159 million, including 3.3 million deaths globally (3).

The $30 \mathrm{~Kb}$ long single-stranded SARS-CoV-2 genome consists of seven genes that are oriented in the following order: ORF1a, ORF1b, S, OEF3, E, M, and N from 5' to 3' direction. The viral mRNA encodes 29 structural and nonstructural proteins (nsps), including ORF 1a/b polyprotein, spike (S) glycoprotein, envelope (E), membrane (M), and the nucleocapsid (N) protein (4). The ORFs $1 \mathrm{a}$ and $1 \mathrm{~b}$ code for the proteases 3Cl-Pro (also known as Mpro or the Main protease), and PL-Pro respectively, that further cleave the polypeptide into 16 nonstructural proteins. These structural proteins play pivotal roles in the entry and assembly of the virus particles in the host cells $[6,7]$. The replication/transcription machinery of the virus is mediated by two enzymes, namely the RNA-dependent R.N.A. polymerase (RdRp) or nsp12 and helicase or nsp13. While RdRP mediates the viral replication, the helicase catalyzes the unwinding of double-stranded RNA formed during replication and allows the next round of viral replication $[8,9]$. Most of these structural and nonstructural proteins of SARS-COV-2 share highly conserved 
functional domains with its predecessor strain SARS-CoV-2(5) and hence may be targeted with the existing or novel antiviral agents (6).

Genomic analysis of the available SARS-CoV-2 sequences across the globe revealed that the viral genome had acquired a specific mutation in S protein, which facilitated its entry and infectivity in host cells. Those variants were found to possess the D614G mutation, i.e. the replacement of the aspartate (D) with glycine (G) at the 614th amino acid of S protein, and became predominant across the globe outcompeting the wild type strain (7). Also, it was further revealed that the D614G mutation enhanced the infectivity of the virus in the host cells $(8,9)$. With the progression of pandemic, SARS-CoV-2 acquired additional mutations in S protein on the background of D614G mutation in different geographical regions and classified as B.1.1.7or N501Y.V1 variant (the UK variant), B.1.351 or N501Y.V2 variant (the South African variant), and P.1 or N501Y.V3 variant (the Brazilian variant). All these three variants possess the mutation in RBD of S protein, characterized by the replacement of asparagine $(\mathrm{N})$ with tyrosine $(\mathrm{Y})$ at position 501 of RBD, on the background of D614G mutation $(10,11)$. The N501Y mutation has been reported to enhance the binding of viral S protein to the host ACE2 receptors and reduce the efficacy of the neutralizing antibodies targeting the $\operatorname{RBD}(12,13)$. This N501Y mutation hence is known as mutation of concern (MOC), and the new SARS-CoV-2 strains harboring this mutation and also additional mutations are termed as the variants of concern (VOC) (14). Thus these accumulating variations had enhanced the immune escape potential of new SARS-CoV-2 variants. Since the prevailing vaccines were designed against the wild-type SARS-CoV-2 discovered in 2019, concerns have been raised about whether these vaccines will be effective against the new VOCs. Hence the search for novel drug candidates which can target both the wild type as well as mutant proteins warrants investigation on utmost priority. Presently there are no well-defined effective therapies against COVID-19. The only medicine prescribed by the doctors is dexamethasone, which has been shown to reduce 28-day mortality in COVID-19 (15). Although Remdesivir, a novel nucleotide analogue, initially showed some promising results in lowering the oxygen requirement of hospitalized COVID-19 patients (16), later WHO has recommended against the use of remdesivir (17). Currently, a plethora of randomized trials are going on to investigate the possible therapeutic remedies against COVID-19, but no conclusive outcome has been found to date.

The beneficial effects of the statin group of drugs are well documented. The statins have shown a promising therapeutic role against various autoimmune inflammatory induced disorders such as multiple sclerosis, systemic lupus erythematosus, rheumatoid arthritis, etc. (18). They also possess anti-hyperlipidemic and cardioprotective properties $(19,20)$. Moreover, statins have also been effective against several viral infections such as Avian influenza, Zika virus, hepatitis C virus, H1N1 pandemic, and also the Ebola outbreak in West Africa (21-25). In an interesting case study published in August 2020, a positive association between statin usage and reduced mortality of COVID-19 patients was first reported (26). It was observed that the antecedent use of statin in hospitalized COVID-19 patients is associated with the lowering of mortality. At a similar time frame, another retrospective case-control study with the COVID-19 patients hospitalized at Hubei province, China, reported that the overall mortality risk had significantly reduced in the patients with in-hospital use of statins, mainly atorvastatin (27). In a retrospective single-center study, Daniels et al. published that statin use before hospitalization was associated with a substantially lower risk of COVID-severity and was also associated with a faster recovery (28). Another multi-centric retrospective cohort study on COVID-19 positive old population living in Belgium revealed a statistically significant association between statin usage and reduced COVID-19-severity (29). In a very recent preprint, Gerold et al. demonstrated the direct effect of statins on coronavirus infection in ex vivo conditions using the human lung cells (30). They observed that among all the statins, fluvastatin significantly reduced the entry of the low pathogenic coronavirus $229 \mathrm{E}$ into the human respiratory cells by modulating the host gene expressions(30). Interestingly, they also observed that fluvastatin treatment significantly decreased SARS-CoV-2 genome copy numbers. But none of the above-mentioned studies had demonstrated the direct effect of statins on SARS-CoV-2 target proteins. Hence the mechanism by which statins may inhibit viral pathogenesis remains inconclusive. In the present study by in silico molecular docking and molecular dynamics simulation analysis of the interactions of the statin group of drugs with the essential structural and functional proteins of SARS-CoV-2, we have aimed to predict a possible mechanism by which the statins may inhibit SARS-CoV-2 infection. 


\section{Materials And Methods}

\section{Selection of target proteins of SARS-CoV-2 and its sequence homology with other coronaviruses}

As discussed earlier, the spike (S)-protein, RNA dependent RNA polymerase (RdRp), 3-Chymotrypsin-like protease (3CL-Pro) or the main protease and helicase are the critical proteins that regulate the various stages of SARS-CoV- 2 infection, including the entry and replication of the viral genome in the host cells $[5-9,26,27]$. Hence for this study, the crystalline structures of RdRp (PDB ID: 7BV2), 3CL-Pro (PDB ID: 6LU7), and helicase (PDB ID: 6ZSL) were obtained from the RCSC Protein Data Bank (https://www.rcsb.org). The S-protein and its two mutants, such as the single mutant having D614G, and the double mutant having D614G/ N501Y, were modeled and subsequently processed via energy minimization (Sect. 2.2).

The FASTA sequences of the target proteins related to SARS-CoV and coronaviruses from other species were derived from UniProt database (https://www.uniprot.org/) for the sequence homology analysis. Multiple sequence alignment studies were performed with Clustal Omega (https://www.ebi.ac.uk/Tools/msa/clustalo/) to get the sequence homology data and generate the phylogenetic tree.

\section{Determination of the mutational landscape of SARS-CoV-2 genome}

SARS-CoV-2 genome sequences deposited to GISAID until April 2021 from the human host were obtained and processed further. After excluding the sequences having an inadequate length ( $<25000 \mathrm{nt}$ ) and duplicated entries, a total of 1299807 sequences were considered for downstream analysis. Sequences were mapped against the SARS-CoV-2 reference sequence (Wuhan/WH01/2019) using NextAlign aligner tool (https://github.com/nextstrain/nextclade). Thereafter, nonsynonymous single nucleotide substitutions in each of the aligned sequences were detected using the Biopython tool. Additionally, to predict the time-dependent emergence of a specific mutation, phylodynamic analysis of global subsamples of SARS-CoV-2 sequences ( $N$ = 3461) was performed using nextstrain/ncov pipeline (https://github.com/nextstrain/ncov). Entropy for each position on SARS-CoV-2 Spike protein, helicase, RdRp and main protease were calculated using a method encoded in the nextstrain/ncov pipeline (https://github.com/nextstrain/ncov).

\section{Homology modeling, energy minimization, and validation of wild type and spike mutants}

The sequence of SARS-CoV-2 spike protein was downloaded from the NCBI protein database (Accession No: YP_009724390). We prepared three sequence files by changing the amino acid in the specified region, i.e. wild type (no alteration), single mutant (D614G), and double mutant (N501Y and D614G). The template sequence was identified via alignment of the available PDB sequences using the BLASTp program (NCBI) for all three types of protein. Due to the unavailability of the crystal structure of the region of interest, we have taken the EM structure as a template (PDB ID: 7BBH) from the PDB database on the basis of query coverage, highest GMQE score, and percent (\%) identity. The structures were then modeled using Swiss Model Server (31) and subjected to further analysis in SWISS-MODEL for the calculation of QMEAN Z-score, which includes cumulative Z-score of $\mathrm{C} \beta$, atomic configuration, solvation, and torsion values. The predictions were finally validated by PROCHECK. The summation of the number of residues in favored regions and in additionally allowed regions was considered for percent (\%) quality assessment using the Ramachandran plot in PROCHECK. For each of the models, energy minimization is done using YASARA protein minimization server (32). After the minimization of the predicted model, all the above parameters were checked. The percentage of the allowed region was given under the Ramachandran plot in Table 1. 
Table 1: Modelling, energy minimization, and structure validation of wild type and

mutant spike proteins

\begin{tabular}{|c|c|c|c|}
\hline $\begin{array}{l}\text { Spike Protein } \\
\text { Model }\end{array}$ & $\begin{array}{l}\text { Structure Quality } \\
\text { after } \\
\text { minimization }\end{array}$ & Tertiary Structure & $\begin{array}{l}\text { Ramacha1 } \\
\text { Plot }\end{array}$ \\
\hline $\begin{array}{l}\text { Wild type } \\
\left(\mathrm{S}_{\mathrm{WT}}\right)\end{array}$ & $\begin{array}{l}\text { QMEAN: }-0.45 \\
\text { C } \beta:-0.32 \\
\text { All atom: }-0.30 \\
\text { Solvation: }-0.96 \\
\text { Torsion:- } 0.07\end{array}$ & & $95.93 \%$ \\
\hline $\begin{array}{c}\text { Single Mutant } \\
\left(\mathrm{S}_{\mathrm{D} 614 \mathrm{G}}\right)\end{array}$ & $\begin{array}{l}\text { QMEAN: }-0.47 \\
\text { C } \beta:-0.29 \\
\text { All atom: }-0.23 \\
\text { Solvation: }-0.91 \\
\text { Torsion:- } 0.12\end{array}$ & 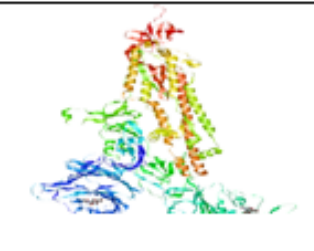 & a \\
\hline $\begin{array}{c}\text { Double Mutant } \\
\left.\text { ( } \mathrm{S}_{\mathrm{D} 614 \mathrm{G} / \mathrm{N5} 01 \mathrm{Y}}\right)\end{array}$ & $\begin{array}{l}\text { QMEAN: }-0.41 \\
\text { C } \beta:-0.10 \\
\text { All atom: }-0.14 \\
\text { Solvation: }-0.82 \\
\text { Torsion:- } 0.12\end{array}$ & $x^{5}+2$ & की \\
\hline
\end{tabular}

\section{Screening of ligand molecules using Schrödinger suite Preparation of the ligand molecules}

Three-dimensional structures of nine statin family of drugs (Atorvastatin, Cerivastatin, Fluvastatin, Lovastatin, Mevastatin, Pitavastatin, Pravastatin, Rosuvastatin, and Simvastatin) were obtained from PubChem database (https://pubchem.ncbi.nlm.nih.gov) (Fig. 2). The physicochemical properties of stains are listed in Table S1. To make the statins suitable for docking, we performed the following steps such as salt removal, the addition of $\mathrm{H}$-atoms, and deprotonation using the LigPrep v4.7 module of the Schrodinger suite. Epik v4.7 module was used for charge neutralization of the drug candidates for attaining the biological relevant $\mathrm{pH}(\mathrm{pH} 7.0 \pm 2)$. The high-energy tautomeric states were excluded to retain up to four stereoisomers, and generation of only one stereoisomer per ligand was allowed. The best drug candidate(s) for different target proteins was predicted by the virtual screening workflow module of Maestro (33).

\section{Preparation of the target proteins}

The target proteins obtained from PDB database or modelled in other platforms required further processing for being used for the docking analysis. The non-standard residues (residues other than amino acids) were first removed from the target proteins using DS client, and the resulted protein structures were processed using the 'protein preparation wizard' of Maestro, Schrodinger (34). The target proteins were processed by the addition of $\mathrm{H}$-atoms, assigning the bond order using CCD. (Cambridge Crystallographic Datacenter) database, and introducing the missing disulfide linkages. The structures were further optimized for H-bond assignment at pH 7.0 using PROPKA function. It was followed by a restrained energy minimization module by applying OPLS3e force field to converge heavy atoms to an RMSD value of $0.30 \AA$ (34).

\section{Grid generation}

The grid generation is a very crucial step for the analysis of drug-target protein interaction. In this process, a 3-D boundary for the ligand-binding site was generated in the target protein using Glide, version 8.2 of Maestro, Schrodinger (35). The top- 
ranked potential binding sites were identified by keeping at least 15 points per site for the generation of the standard grid. The 'receptor grid generation' module was used to generate grids of $20 \AA$ size across the binding site.

\section{Molecular Docking}

The docking of the statin molecules with the target proteins was performed in three steps, namely, high throughput virtual screening (HTVS), standard precision (SP), and extra precision (XP). The processed ligands (statins) were first screened for their binding affinities to the target proteins using the High-Throughput-Virtual Screening (HTVS) module (36). Then Flexible docking and subsequent post docking minimization were performed to keep $25 \%$ of the best compounds (retaining all states, having high or low scoring values). After HTVS, statins were subjected to Standard Precision (SP) docking (flexible) followed by extra precision (XP) docking (flexible). The parameters such as Glide score, Glide energy, Glide emodel, and Glide ligand efficiency were detected, and $25 \%$ of the compounds with the best scoring state were retained. Post docking binding-site analysis and generation of graphical representations were done with Discovery Studio Client, version 16.10 (Accelrys Software Inc. San Diego) (37) and V.M.D. (Visual Molecular Dynamics) $(38,39)$ software.

\section{Molecular Dynamics Simulations}

Molecular dynamics (MD) simulations provided an insight into dynamic perturbations within the complex and interactions of ligand, lipid, and water molecules. The MD simulation studies of free protein templates and protein-ligand complex were performed using NAMD (Nanoscale Molecular Dynamics) program; v 2.8 (40), Amber10: EHT forcefield (41), and generalized Born Implicit solvent model was applied (42). The system was heated from $300 \mathrm{~K}$ to $310 \mathrm{~K}$ for $100 \mathrm{ps,}$, and then equilibrated at $310 \mathrm{~K}$ for $100 \mathrm{ps}$, followed by the production stage for $2 \mathrm{~ns}$, and finally cooling to $300 \mathrm{~K}$ for $100 \mathrm{ps}$. The equation of motion was discretized at a $2 \mathrm{fs}$ interval. Here, Langevin dynamics model $(43,44)$ was used for the proposed molecular dynamics simulation described above. Frames were collected at 0.5 ps interval. The trajectory files were analyzed with VMD. (38) and VEGA ZZ (45) for generating the data of RMSD, RMSF, SASA, and radius of gyration plot. The radius of gyration is a significant factor for the structural integrity of the crystal structure of protein and protein-ligand complexes. Here, the student t-test was analyzed for statistical evaluation (times $=2 \mathrm{fs}$; for 4600 frames), and the results were statistically significant $(p<$ 0.0001). Solvent accessible surface area (SASA) is a measurement of the surface area of the ligand that is accessible to solvent molecules and also referred to as PSA (Polar solvent accessible area). SASA versus MD simulation time plots were used throughout the study to investigate changes in the position and conformation of the ligands within the protein complex corresponding through 4600 frames (for $2 \mathrm{~ns}$ ). Moreover, the gyration radius ( $\mathrm{rg}$ ) and solvent accessible surface area (SASA) for the free protein and statin-protein complexes were calculated. The radius of gyration describes the overall spread of the molecule and is defined as the root mean square distance of the collection of atoms from their common centre of gravity. SASA represents the solvent accessable surface area of the protein and determines wheather the amino acid residues are buried or exposed.

\section{Results}

\section{Evolutionary relationship of therapeutically targeted SARS-CoV-2 proteins with other viral strains}

The evolutionary conservancy is a critical feature of a gene or protein which guides it to act as a potential drug target. Hence the determination of interspecies evolutionary distance is necessary to predict the effectiveness of the candidate protein to become a potential drug target. In this study, we investigated the interspecies divergence of four selected target proteins of SARS-CoV-2, namely the spike protein, RdRp, helicase, and main protease among the Coronavirinae family members (Fig. S1). Protein sequence (FASTA) alignment of SARS-CoV-2 proteins with other coronavirus species from bat, civet, pangolin, including SARS-CoV were performed using P-BLAST. The analysis showed high sequence similarity for RdRp (>95\%), helicase (>99\%), and main protease (>95\%) proteins (Fig. S1). SARS-CoV-2 spike (S) protein showed maximum sequence homology with pangolin coronavirus (92.43\%) and over 76\% homology with coronaviruses from other species. 


\section{Mutational landscape of the SARS-CoV-2 genome}

From 1299806 SARS-CoV-2 genome sequences deposited to GISAID database till April 2021, we have detected 18637447 nonsynonymous single nucleotide substitutions in 9742 nucleotide positions of $\sim 30 \mathrm{~Kb}$ viral genome. Among these, about 35 sites were mutated in more than $5 \%$ of sequences, and the rest were nonrecurrent. The recurrent mutations mainly comprise alterations in spike protein (9 mutations) and nucleocapsid protein (9 mutations) (Fig. S2). The most frequent spike protein mutations that are predominant to date were D614G (95.71\%), P681H (38.61\%), and N501Y (37.70\%) (Fig. 1A). It was observed that the spike D614G mutation became predominant $(\sim 70 \%)$ since March 2020 , just within two months of its origin, both $\mathrm{P} 681 \mathrm{H}$ and N501Y started gaining frequency from December 2020 ( 17-18\%) and achieved 50\% frequency in two months down the line. Both these mutations occurred in the background of the D614G mutation. Previous studies have already shown the functional impact of spike D614G and N501Y mutations that provided a selective advantage to these mutations in viral entry, either through the interaction of ACE2 or by S1-S2 cleavage $(9,46-48)$. Moreover, the N501Y mutation in S protein is present in more than $90 \%$ of sequences of B.1.1.7 variant (the UK variant), variant (the South African variant), and P.1 variant (the Brazilian variant) and exhibited stronger interaction with the host ACE-2 receptor(49). Hence it is also known as the MOC or mutation of concern.

Additionally, to remove biases due to oversampling of SARS-CoV-2 sequences from a specific geographic region, a phylodynamic time tree analysis from a global subsample of 3461 SARS-CoV-2 sequences was performed (Fig. 1B). Phylodynamic analysis showed, 84-positions on SARS-CoV-2 genome to possess entropy (i.e. nucleotide diversity) over 0.1 and only 10-positions harboured entropy over 0.5 [3-positions on nucleocapsid (entropy: 0.52-0.76), 2-positions on spike (0.61-0.62), 1-position in ORF3a (0.61) and 4-positions on ORF1a (0.51-0.59)]. The viral diversity across sampled sequences across the world showed a similar pattern at the time of analysis. These results further highlight that a single drug target for any of these proteins may be applied to different SARS-CoV-2 lineages that are in circulation throughout the world.

\section{Homology modeling, refinement, and structure validation of wild type and mutant S proteins}

The template sequence for modeling the wild type and spike mutants was identified through searching on PDB using the BLASTp program. The BLAST search against the PDB database provided homology sequences against several structures. The PDB 7BBH showed maximum query coverage (96\%), highest GMQE score (0.71), and present identity (89\%), along with the resolution $(2.90 \mathrm{~A})$ of the structure. Hence $7 \mathrm{BBH}$ was selected as the template structure for the modeling of wild-type and mutant S proteins. Also, the GMQE score is generated by the target-template alignment of the modeled protein in SWISSMODEL, and the value is expressed as a number between zero and one. Higher GMQE scores indicate increased structural reliability. The GMQE score for the modeled protein was found to be 0.98 , which indicates good model accuracy. We prepared three sequence files by changing the amino acid in the specified region, i.e. wild type (no alteration), single mutant (Aspartate at 614th position altered to Glycine-S ${ }_{\mathrm{D} 614 \mathrm{G}}$, and double mutant (Aspartate at 614th position altered to Glycine-

$\mathrm{S}_{\mathrm{D} 614 \mathrm{G}}$ and Asparagine at 501st position changed to Tyrosine- $\left.\mathrm{S}_{\mathrm{D} 614 \mathrm{G} / \mathrm{N} 501 \mathrm{Y}}\right)$. After the modeling and refinement, each structure was validated through the generation of the Ramachandran plot using the PROCHECK tool (Table 1).

Ramachandran plot confirmed that most of the amino acids of the modeled structures lie in the allowed region (>95\%), which indicates the stability of the structures (Table 1).

\section{Molecular docking of the target proteins with statins RNA dependent RNA polymerase (RdRp)}

The cryo-EM structure of SARS-CoV-2 RdRp bound to the drug remdesivir at nsp 12 was first deduced by Yin. et al. in 2020 (50), where they reported the existence of the complex either in the apo form or in a complex with the primer RNA (PDB ID: 7BV2). The structural features of RdRp revealed that the polymerase domain consisted of a 'finger' domain (amino acid 
residues: 398-581, 628-687), a 'palm' domain (amino acid residues: 582-627, 688-815), and a 'thumb' domain (amino acid residues: 816-919) and also an additional nidovirus-unique N-terminal extension (amino acid residues: 1-397) [32]. As per PDBsum record, 7BV2 has seven beta-sheets, forty-six a - helices, fifty-eight beta-turns, and two gamma-turns. 7BV2 comprises six active sites such as AC1, AC2, AC3, AC4, AC5, and AC6, respectively, of which AC3 and AC6 constitute the remdesivir binding site.

Molecular docking of 7BV2 with nine statin molecules revealed that only fluvastatin, pitavastatin, pravastatin, rosuvastatin, and simvastatin qualified with a docking score while the other statin molecules did not qualify the screening (Table 2). Among those statins, fluvastatin and pitavastatin were the best candidate molecules with higher binding affinities (Fig. 3, Fig. S3 and Fig. S4, Table 2). In contrast, the other statin molecules did not exhibit strong binding (Table 2). The 3D structures of RdRp-ligand complexes and their binding sites were shown in Fig. 3 and Fig. S3-S4 (A\&B). Both the molecules bind at the remdesivir binding site of the enzyme. The glide score, emodel score, and energy values for fluvastatin were $7.441,-60.23$, and $-57.076 \mathrm{kcal} / \mathrm{mol}$ (Table 2), and the corresponding values for pitavastatin were $-7.5,-62.341$, and $58.654(\mathrm{kcal} / \mathrm{mol})$, respectively (Table 2$)$. In our study, we observed that the glide score, emodel score, and energy values for remdesivir were $-7.312,-56.672$, and $-71.38 \mathrm{kcal} / \mathrm{mol}$, respectively. 
Table 2

Docking results of the statin molecules to SARS-CoV-2 target proteins

\begin{tabular}{|c|c|c|c|c|c|c|c|c|}
\hline \multirow[t]{2}{*}{ Target Protein } & \multirow[t]{2}{*}{ PDB ID } & \multirow{2}{*}{$\begin{array}{l}\text { Selected } \\
\text { ligands }\end{array}$} & \multirow{2}{*}{$\begin{array}{l}\text { glide } \\
\text { score } \\
\text { (kcal/mol) }\end{array}$} & \multirow{2}{*}{$\begin{array}{l}\text { glide } \\
\text { energy } \\
(\mathrm{kcal} / \mathrm{mol})\end{array}$} & \multirow{2}{*}{$\begin{array}{l}\text { glide } \\
\text { emodel } \\
\text { (kcal/mol) }\end{array}$} & \multicolumn{3}{|c|}{ Centre of binding location } \\
\hline & & & & & & $\mathrm{X}$ & $\mathrm{Y}$ & Z \\
\hline \multirow[t]{5}{*}{$\mathrm{RdRp}$} & \multirow[t]{5}{*}{ 7BV2 } & Fluvastatin & -7.441 & -57.076 & -60.23 & 84.51 & 100.41 & 109.24 \\
\hline & & Pitavastatin & -7.5 & -58.654 & -62.34 & 85.11 & 100.70 & 109.73 \\
\hline & & Pravastatin & -6.03 & -38.82 & -47.42 & - & - & - \\
\hline & & Rosuvastatin & -5.93 & -39.29 & -44.43 & - & - & - \\
\hline & & Simvastatin & -4.59 & -27.09 & -29.21 & - & - & - \\
\hline \multirow[t]{6}{*}{ 3CL-Pro } & \multirow[t]{6}{*}{ 6LU7 } & Fluvastatin & -7.338 & -48.617 & -61.748 & -10.37 & 15.56 & 68.24 \\
\hline & & Pitavastatin & -7.119 & -45.785 & -57.563 & -11.48 & 13.49 & 69.05 \\
\hline & & Cerivastatin & -5.2 & -36.36 & -46.71 & - & - & - \\
\hline & & Atorvastatin & -4.9 & -50.73 & -58.99 & - & - & - \\
\hline & & Rosuvastatin & -4.1 & -29.65 & -34.64 & - & - & - \\
\hline & & Simvastatin & -3.5 & -26.18 & -27.54 & - & - & - \\
\hline \multirow[t]{6}{*}{ Helicase } & \multirow[t]{6}{*}{$6 Z S L$} & Fluvastatin & -11.333 & -58.72 & -66.511 & -20.68 & 33.63 & -25.84 \\
\hline & & Pravastatin & -7.612 & -48.408 & -55.673 & -10.10 & 28.20 & -51.15 \\
\hline & & Atorvastatin & -5.99 & -35.89 & -46.18 & - & - & - \\
\hline & & Pitavastatin & -5.99 & -39.26 & -56.94 & - & - & - \\
\hline & & Cerivastatin & -5.7 & -40.33 & -52.88 & - & - & - \\
\hline & & Rosuvastatin & -4.8 & -32.94 & -33.07 & - & - & - \\
\hline \multirow[t]{2}{*}{ Spike $_{W T}$} & \multirow{2}{*}{$\begin{array}{l}7 \mathrm{BBH} \\
\text { (modelled } \\
\text { protein) }\end{array}$} & Fluvastatin & -5.856 & -38.632 & -55.522 & 277.91 & 235.61 & 240.32 \\
\hline & & Cerivastatin & -5.305 & -41.464 & -47.432 & - & - & - \\
\hline \multirow[t]{4}{*}{ Spike $_{\text {D614G }}$} & \multirow{4}{*}{$\begin{array}{l}7 \mathrm{BBH} \\
\text { (modelled } \\
\text { protein) }\end{array}$} & Fluvastatin & -7.380 & -47.041 & -68.161 & 238.78 & 201.88 & 202.691 \\
\hline & & Cerivastatin & -7.114 & -46.204 & -64.639 & - & - & - \\
\hline & & Rosuvastatin & -5.972 & -37.104 & -43.851 & - & - & - \\
\hline & & Pravastatin & -4.950 & -35.271 & -35.417 & - & - & - \\
\hline \multirow[t]{4}{*}{ Spike $_{\text {D614G/N501Y }}$} & \multirow{4}{*}{$\begin{array}{l}7 \mathrm{BBH} \\
\text { (modelled } \\
\text { protein) }\end{array}$} & Fluvastatin & -7.811 & -45.207 & -63.749 & 236.24 & 201.63 & 203.586 \\
\hline & & Pitavastatin & -7.223 & -42.819 & -59.011 & - & - & - \\
\hline & & Rosuvastatin & -6.925 & -48.802 & -66.427 & - & - & - \\
\hline & & Cerivastatin & -6.221 & -39.811 & -57.555 & - & - & - \\
\hline
\end{tabular}

The binding pockets for fluvastatin and pitavastatin were further analyzed using the Discovery Studio client. The fluvastatin binding pocket was found to be hydrophilic in nature (Fig. S3C) due to the presence of hydrophilic amino acids like ASN 496, ARG 569, ASN 497, SER 501, THR 565, ASN 543 and SER 561. Since, except arginine, all other hydrophilic amino acids are uncharged, the binding pocket is nearly neutral (Fig. S3D). The presence of both the H-bond donor residues (ASN 496 and 
ARG 569) and H-bond acceptor residues (GLY 683) has made this site favorable for the formation of $\mathrm{H}$-bond with the ligand (fluvastatin). These residues, present in 32nd and 47th $\beta$-turn formed conventional H-bonds with the carboxyl and hydroxyl groups of fluvastatin (Fig. 3B). Moreover, the alkyl group of fluvastatin had hydrophobic interactions with the VAL 557 residue (Fig. 3B). All these interactions are favorable for forming a stable fluvastatin-RdRP complex.

The calculated LogP and pKa values of fluvastatin and pitavastatin are similar (LogP: 4.85 and 4.8; pKa: 4.5 and 4.3) (Table S1). Hence the binding pocket for pitavastatin showed similar properties to the fluvastatin-binding pocket. The presence of uncharged polar amino acids like SER 501, ASN 543, ASN 497, THR 565, and positively charged basic amino acids like ARG 569, LYS 500, and ARG 555 contribute to the hydrophilicity of the pitavastatin binding pocket. In contrast, the presence of uncharged nonpolar amino acids like VAL 557, ILE 562, VAL 560, VAL 557, ALA 512, ALA 558, and ALA 502 contribute to the hydrophobicity of the binding pocket (Fig. S4C). The presence of basic amino acids imparts a slight basic character to the binding pocket of pitavastatin to RdRp (Fig. S4D). The carboxylic acid group and hydroxyl groups of pitavastatin form $\mathrm{H}$ bonds with the $\beta$-turn (32nd and 33rd) and 27th helical region of the protein. SER 501 and VAL 560 act as H-bond acceptors, whereas ARG 569 is H-bond act as the donor (Fig. 3D). The negatively charged O-atom (formed as a result of ionization of $\mathrm{COOH}$ group) formed salt-bridge with ARG 569. The $\pi$ - electron cloud of the fluorophenyl group interacted with alkyl groups $\left(-\mathrm{CH}_{3}\right)$ of VAL 557, located in the 14th $\beta$ strand of D sheet, and formed $\pi$ - alkyl bond. The electron cloud of the quinoline ring contributed to interaction with GLY 683 residue, located in the 35th $\beta$ strand of the $D$ sheet. All these interactions contribute to a stable interaction of pitavastatin with RdRp.

Thus the results indicate that both Fluvastatin and Pitavastatin have a strong binding affinity towards RdRP and bind at the remdesivir binding pocket of the enzyme, and these two statins may inhibit enzyme activity.

\section{CL-Pro or M-protease}

As per PDBsum record, 3 CL-Pro (306 amino acid long, PDB ID: 6LU7) has a single chain consisting of two beta-sheets, ten helices, twenty-six beta-turns and three gamma turns. A previous report by Jin. et al. (2020) demonstrated that the enzyme consists of an active site to which the inhibitors can bind and inhibit the enzyme activity (51). The inhibition site comprised of the amino acids such as HIS 41, MET 49, TYR 54, PHE140, LEU 141, ASN 142, GLU 166, HIS 163, MET 165, MET 165, LEU 167, HIS 172, PHE 185, and GLN 192 and the natural compounds lime Ebselen, Disulfiram, Tideglusib, Carmofur, Shikonin and PX-12 were reported to bind at that site (51). Recently, it was reported that pitavastatin and fluvastatin can bind to 3-CLPro with the binding energy of -8.2 and $-7.7 \mathrm{kcal} / \mathrm{mol}$ (52), but the report lacks detailed docking analysis and dynamics studies to support the prediction.

Molecular docking of the statins with 6LU7 revealed that fluvastatin and pitavastatin are the best ligands to bind at the active site (site of inhibition). In contrast, the other statin molecules did not exhibit strong binding (Table 2, Fig. 4). The detailed analysis of ligand-protein complexes are represented in Fig. 4A-B \& Fig. S5 (for fluvastatin) and Fig. 4C-D \& Fig. S6 (for pitavastatin). The values of glide score, emodel, and glide energy for fluvastatin were $-7.338,-61.748$, and -48.617 $(\mathrm{kcal} / \mathrm{mol})$, respectively, whereas the same for pitavastatin were $-7.119,-57.563$, and $-45.785(\mathrm{kcal} / \mathrm{mol})$, respectively (Table 2). Thus the data obtained from the docking analysis indicated the strong affinity of fluvastatin and pitavastatin towards the enzyme.

Characterization of the binding cavity of fluvastatin revealed that it is partly hydrophilic (Fig. S5C, Blue color). The binding cavity is comprised of the residues like THR 25, THR 26, TYR 54, ASP 187, and GLN 189, which impart a polar (hydrophilic) character to the binding pocket (Fig. S5C). As per ionizibility, the binding site mainly was neutral due to the presence of the residues like THR 26, HIS 41, TYR 54, and CYS 145 (fade white color, Fig. S5D), while only GLU 166 was acidic (slightly brown in color, Fig. S5D). The presence of H-donor amino acid residues (THR 26, HIS 41, TYR 54, and CYS 145) and Hacceptor amino acid residue (GLU 166) results in H-bond formation with fluvastatin. Additionally, the fluorine atom of

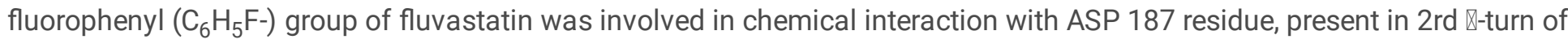
the protein, while the 0 -atoms of polyhydroxy groups $(-\mathrm{OH})$ interacts with the HIS 41 residue, present in 2nd helices. 
Moreover, the alkyl group of fluvastatin had hydrophobic interactions with CYS 145 and HIS 163, present on the 12th $\beta$ strand of $B$ sheet and 17th $\beta$ turn, respectively (Fig. 4B). All these interactions contributed to a stable fluvastatin-3CL-Pro complex.

Due to the similar LogP values (Table S1) the binding pocket for pitavastatin is similar to that of fluvastatin. The binding site of pitavastatin was slightly basic due to the occurrence of the basic amino acid residues like HIS 41, HIS 163, HIS 164, HIS 141, and ARG 188 (Fig. S 6D). HIS 41 acts as H-donor while GLU 166 and ARG 188 act as H-acceptor and participate in the formation of conventional H-bond with hydroxyl groups of pitavastatin (Fig. S6E). The electron cloud of the fluorophenyl group of pitavastatin contributed to $\pi-\pi$ stacked interaction with LEU 141, present in 16th $\beta$ turn of the protein (Fig. 4D). All these interactions contribute to the formation of a stable pitavastatin - 3CL-Pro complex.

\section{Helicase}

Helicase (Nsp13) is a multi-functional protein that consists of $\mathrm{N}$-terminal $\mathrm{Zn}$-binding domain (ZBD) and the helicase domain $(\mathrm{Hel})$. The $\mathrm{N}$-terminal region consists of twenty-six cysteine residues that form the $\mathrm{Zn}^{2+}$ binding domain, whereas the helicase domain comprises a conserved motif at the C-terminus(53). Helicase requires ATP hydrolysis for its action, and the residues such as SER 310, LYS 288, and GLU 375 constitute the ATP-binding site or the active site(54). The crystal structure of the SARS-CoV-2 helicase (resolution $1.94 \AA$ ) was reported by Newman J. A. et al. (PDB ID: 6ZSL) (https://www.rcsb.org/structure/6ZSL). As per PDBsum record, helicase consists of nine beta-sheets, thirty-four beta-strands, twenty-three helices, forty-four beta turns, and three gamma-turns.

Molecular docking of 6ZSL with the statins revealed that fluvastatin exhibited the strongest binding affinity with the glide score, emodel, and glide energy values of $-11.333,-66.511$ and $-58.72(\mathrm{kcal} / \mathrm{mol})$, respectively (Table 2). The 3D structure of the fluvastatin-helicase complex is shown in Fig. $5 \mathrm{~A}$ and $5 \mathrm{~B}$, while the major $2 \mathrm{D}$ interactions are shown in Fig. $5 \mathrm{C}$. Further characterization of the fluvastatin-binding site on helicase revealed the hydrophilic amino acid residues (GLY 265, THR 286, GLY 287, LYS 288, HIS 290, SER 310, ARG 443, GLN 527, ASP 374, GLU 375, and SER 539) outnumbered the hydrophobic amino acid residues (PRO 283, PRO 284, ALA 312, ALA 313, and ALA 316) to make the pocket preferentially hydrophilic (Fig. 5D-5F). The pH profile of the binding cavity revealed that the region having ASP 374 and GLU 375 residues is acidic, whereas ARG 443, LYS 288, and HIS 290 rich area is basic (Fig. 5D). The ionized form (negatively charged) of fluvastatin was in proper pose to form 2 salt bridges between the $-\mathrm{COO}^{-}$group and basic amino acids LYS 288 of 8 th helices, ARG 443 of $41 \mathrm{st} \beta$-turn. The binding cavity is rich with both the $\mathrm{H}$-bond donor and acceptor residues which facilitate the formation of multiple $\mathrm{H}$-bonds with fluvastatin (Fig. 5E). The negatively charged O-atom of - $\mathrm{COO}^{-}$group of fluvastatin formed $\mathrm{H}$-bond with GLY 285, whereas the carbonyl O-atom formed H-bond with GLY 287, and LYS 288. The LYS 288 residue formed an additional $\mathrm{H}$-bond with the $-\mathrm{OH}$ group attached to the aliphatic chain. Moreover, ten hydrophobic interactions, such as alkylalkyl, pi-alkyl, pi-sigma, amide-pi stacked, were observed between the $\pi$ electron-rich aromatic rings of fluvastatin and the surrounding amino acid residues like GLY 538, ALA 312, ALA 313, and ALA 316. All these interactions had synergistic effects to make helicase the preferable target protein for fluvastatin. Interestingly, the previous study had revealed that the ATP binding site of helicase is comprised of amino acid residues like K288, E375, Q404, R443, and R567 (55). In our study, we have observed that fluvastatin binds to helicase in a similar region, and the residues like LYS 288 and ARG 443 play important roles in this interaction (Fig. 5B \& Fig. S7). Hence it may be concluded that fluvastatin may interfere with the ATP binding site of helicase and inhibit the activity of the enzyme.

\section{Wild type (Spike $\left.{ }_{\mathrm{wt}}\right)$ and mutant spike proteins (Spike ${ }_{\mathrm{D} 614 \mathrm{G}}$ and spike $_{\mathrm{D} 614 \mathrm{G} / \mathrm{N} 501 \mathrm{Y})}$}

Spike protein is one of the essential proteins required for the entry of SARS-CoV2 into the host cell. The viral entry is mediated by the interaction of the RBD (receptor binding domain) of S1 subunit of spike with the ACE2 receptor of the host cell membrane is mainly responsible for the entry of SARS-CoV2 into the host cell. The structural insights of the different 
conformational states of the S-protein and S1(RBD)-ACE2 complex have been reported (56). Further S1- subunit is composed of four domains, namely the N-terminal domain (NTD), RBD, and two C-terminal domains (CTDs), CTD-1, and CTD-2 [45]. In our modeled S-proteins (wild type S protein and the mutants), the amino acids 14 to 685 constitute the S1 region, while the residues 686 to 1273 comprise the S2 site, which corroborates with the published report (57).

Molecular docking of the wild-type S-protein with the statins revealed that fluvastatin exhibited the highest binding affinity to

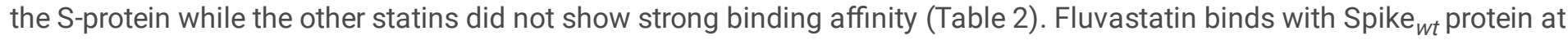
NTD. (N-Terminal Domain) region (the 3D structure is shown in Fig. 6A and 2D interaction diagram is shown in Fig. 6B), with the glide score and glide energy were -5.854 and $-38.632 \mathrm{Kcal} / \mathrm{mole}$, respectively (Table 2). The binding cavity of fluvastatin on S-protein was composed of hydrophilic residues like SER 98, THR 259, HIS 245, and LYS 97; and hydrophobic amino acid residues like TYR 144, TRP 258, ALA 260, and ALA 262 (Fig. S8C). The presence of HIS 245, TYR 144, and LYS 97 shifted the $\mathrm{pH}$ of the binding pocket to a slightly basic range that is suitable for the interaction of ionized state of fluvastatin (Fig. S8D). The negatively charged O-atom of $-\mathrm{COO}^{-}$formed a salt bridge with LYS 97 . Though the surface of the binding pocket is rich with $\mathrm{H}$-bond donor and $\mathrm{H}$-bond acceptor regions (Fig. S8E), no suitable pose of fluvastatin was found to have $\mathrm{H}$-bond with the carbonyl O-atom. The two hydroxyl groups attached to the aliphatic chain worked as the acceptor residue to form conventional H-bond with SER 98 and ALA 262. The $\pi$-electron cloud of the benzene ring of fluvastatin formed a pi-pi stacking bond with HIS 245, whereas the electron-rich indole ring did not show any hydrophobic interactions. Compared to the other target proteins, only fewer interactions were observed when fluvastatin was docked with the modelled S-protein. This might justify the relatively lower affinity of fluvastatin to Spike $\mathbf{w t}_{\mathrm{wt}}(-5.854 \mathrm{Kcal} / \mathrm{mole})$.

The result of the molecular docking study of fluvastatin to single mutant spike ${ }_{\mathrm{D} 614 \mathrm{G}}$ was shown in Fig. 6C-6D \& Fig. S9. The docking analysis revealed that the binding site of fluvastatin on spike ${ }_{D 614 G}$ was altered than that on the wild-type protein. Fluvastatin binds to N-terminal domain of Spike ${ }_{\mathrm{wt}}$, but in spike $_{\mathrm{D} 614 \mathrm{G}}$ it binds at the S2 subunit (Fig. 6C-6D). The binding site was found to be rich in hydrophobic amino acid residues like ILE 850, ILU 959, ALA 956, VAL 952, ILE 834, PHE 833, VAL 860, LEU 861, PRO 862, PRO 863, ALA 829, and LEU 828, which imparts a hydrophobic character to the binding site (Fig. S9C). Additionally, the polar amino acids like ASN 955, THR 732, HIS 1058; the basic amino acids like LYS 854 and LYS 835; and the acidic amino acid ASP 830 were also present, contributing to the hydrophilicity of the binding pocket. The pH was favorable for docking of fluvastatin molecules at a negatively charged state (Fig. S9D). The negatively charged O-atom of $\mathrm{COO}^{-}$of fluvastatin formed salt bridges with LYS 835 and LYS854. The presence of both anionic and cationic amino acid residues made the pocket surface rich with $\mathrm{H}$-bond acceptor and $\mathrm{H}$-bond donor points (Fig. S9E). The carbonyl O-atom acted as the receptor to form $\mathrm{H}$-bond with LYS 854. The aliphatic hydroxyl groups of fluvastatin formed two conventional $\mathrm{H}$-bond with ASP 830 and VAL 860. Moreover, the pose of fluvastatin was suitable to form multiple hydrophobic interactions (alkylalkyl, and pi-alkyl) between the electron cloud of aromatic rings and amino acid residues like LEU 828, ALA 829, ALA 956. Thus, the docking site at Spike $_{\mathrm{D} 614 \mathrm{G}}$ is more favorable for a higher number of noncovalent interactions compared to Spike $_{\mathrm{wt}}$. Therefore, the binding affinity was significantly higher, as reflected with the calculated glide score $(-7.378 \mathrm{kcal} / \mathrm{mol})$ and glide energy $(-47.041 \mathrm{kcal} / \mathrm{mol})$ (Table 2).

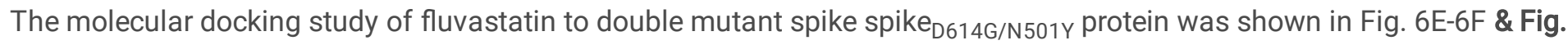
$\mathrm{S} 10$. Analysis of the binding site revealed that fluvastatin binds at the $\mathrm{S} 2$ region of spike $\mathrm{D}_{\mathrm{D} 14 \mathrm{G} / \mathrm{N} 501 \mathrm{Y}}$ protein. The binding pocket was found to be rich in hydrophobic amino acid residues like ILE 850, VAL 860, PRO 862, PRO 863, LEU 959, ALA 956, VAL 952, LEU 828, and ALA 829, which imparts a hydrophobic character to the binding site (Fig. S10C). But polar amino acids like ASN 955, THR 732, HIE 1058, GLN 853, THR 859; basic acid amino acids like LYS 854, LYS 835; and acidic amino acid ASP 830 contributed to the hydrophilicity of the binding pocket. The $\mathrm{pH}$ was suitable for docking of fluvastatin in a negatively charged state (Fig. S10D). The O-atom of the carboxylic group (-COOH) of fluvastatin formed a salt bridge with LYS854. The presence of both anionic and cationic amino acid residues made the pocket rich with $\mathrm{H}$-bond acceptor and $\mathrm{H}$ bond donor points (Fig. S10E). The carbonyl 0-atom formed H-bond with LYS 854. Additionally, the aliphatic hydroxyl groups formed H-bond with VAL 860 and ASP 830. Moreover, the F-tom of fluvastatin developed an H-bond with GLN 853. It also 
interacted with ALA 956. Additionally, the pose of fluvastatin was suitable to form hydrophobic interactions like alkyl-alkyl, pi-alkyl between the electron cloud of aromatic rings and ILE 850, ALA 829, and LEU 828. All these interactions had

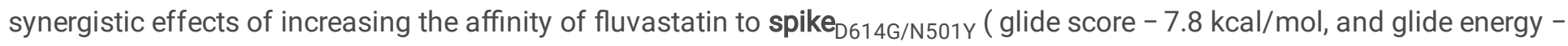
$45.217 \mathrm{kcal} / \mathrm{mole}$ ) in comparison to Spike $\mathrm{wt}_{\mathrm{wt}}$ and Spike ${ }_{\mathrm{D} 614 \mathrm{G}}$ (Table 2).

\section{Molecular dynamics simulation of Fluvastain-target protein complexes}

To validate the conformational changes of the target proteins in the free state or in complex with the statins, we performed two nanoseconds of MD simulation using NAMD (Nanoscale Molecular Dynamics) program; $v$ 2.8. The parameters such as RMSD, RMSF, SASA, and radius of gyrations were analyzed throughout the simulation trajectory to determine the stability of the drug-protein complex. The docking analysis demonstrated that among all the nine statin molecules, fluvastatin showed a fair, binding affinity to the target proteins, and the highest affinity was observed for the helicase protein. Hence we have performed the MD simulation for fluvastatin-target protein complexes.

\section{Helicase-Fluvastatin complex}

The molecular dynamic simulation analyses of the fluvastatin-helicase complex were shown in Fig. 7A-7D and summarized in Table 3. The RMSD plot (Fig. 7A) indicated that the complexation with fluvastatin did not compromise the stability of the helicase protein. Rather, the fluvastatin-helicase complex had RMSD (3.867 \pm 0.007232$)$ significantly less than that of free helicase. The poses of fluvastatin at the beginning and end of the simulation period have been superimposed in Fig. 7B. The RMSD value was $0.7272 \AA(<2 \AA)$, suggesting the stability of fluvastatin at the docking site. The comparative RMSF plot of free helicase and fluvastatin-helicase complex indicated some fluctuations in the E1 and E2 region of the protein (color ballot )(E1 and E2), with no significant change in the region comprising of the Fluvastatin binding site ( $285-550$ residues) (Fig. 7C \& 7D). Moreover, upon complexation with fluvastatin, the average RMSF value helicase was reduced from $1.096 \pm$ $0.0113 \AA$ to $1.031 \pm 0.0128 \AA$ respectively, which indicates the formation of a thermodynamically favourable complex. Moreover, The SASA value was reduced from $43091 \pm 2.887 \AA^{2}$ for the free protein to $38259 \pm 2.827 \AA^{2}$ in the fluvastatinhelicase complex without any major change in the gyration radius $\left(r_{g}\right)$ (Fig. S11A, S11B). These results indicate a strong interaction of fluvastatin with the helicase without any unfolding of the protein structure. 
Table 3

Summary of Molecular dynamics studies of the fluvastatin-target protein complex.

\begin{tabular}{|c|c|c|c|c|c|}
\hline SI.No & $\begin{array}{l}\text { Target } \\
\text { Protein }\end{array}$ & $\begin{array}{l}\text { Average RMSD } \\
(\AA)\end{array}$ & Average RMSF ( $(̊)$ & $\begin{array}{l}\mathrm{r}_{\mathrm{g}} \\
(\AA)\end{array}$ & $\begin{array}{l}\text { SASA } \\
\left(\AA^{2}\right)\end{array}$ \\
\hline 1. & Free-RdRp & $\begin{array}{l}3.713 \pm 0.006144 \\
n=4600 \text { frames }\end{array}$ & $\begin{array}{l}0.9124 \pm 0.01247,(\text { amino } \\
\text { acid residues }=1157)\end{array}$ & $\begin{array}{l}29.59 \pm 0.002613 \\
n=4600\end{array}$ & $\begin{array}{l}38565 \pm 2.3, n= \\
4600 \text { frames }\end{array}$ \\
\hline 2. & $\begin{array}{l}\text { Fluvastatain-RdRp } \\
\text { complex }\end{array}$ & $\begin{array}{l}2.584 \pm 0.00357 \\
\mathrm{n}=4600 \text { frames }\end{array}$ & $\begin{array}{l}0.841 \pm 0.009764,(\text { amino } \\
\text { acid residues }=1157)\end{array}$ & $\begin{array}{l}29.89 \pm 0.001316 \\
n=4600 \text { frames }\end{array}$ & $\begin{array}{l}33766 \pm 2.0, n= \\
4600 \text { frames }\end{array}$ \\
\hline 3. & Free-3CL-Pro & $\begin{array}{l}2.889 \pm 0.005321 \\
n=4600 \text { frames }\end{array}$ & $\begin{array}{l}0.8456 \pm 0.01703,(\text { amino } \\
\text { acid residues }=306)\end{array}$ & $\begin{array}{l}21.31 \pm 0.02369 \\
n=4600 \text { frames }\end{array}$ & $\begin{array}{l}11661 \pm 10.9, n \\
=4600 \text { frames }\end{array}$ \\
\hline 4. & $\begin{array}{l}\text { Fluvastatain- 3CL- } \\
\text { Pro complex }\end{array}$ & $\begin{array}{l}2.577 \pm 0.004656 \\
n=4600 \text { frames }\end{array}$ & $\begin{array}{l}0.7794 \pm 0.01598,(\text { amino } \\
\text { acid residues }=306)\end{array}$ & $\begin{array}{l}21.6 \pm 0.01411, n \\
=4600\end{array}$ & $\begin{array}{l}11767 \pm 8.8, \mathrm{n}= \\
4600 \text { frames }\end{array}$ \\
\hline 5. & Free-Helicase & $\begin{array}{l}4.231 \pm 0.00664 \\
n=4600 \text { frames }\end{array}$ & $\begin{array}{l}1.096 \pm 0.01134,(\text { amino } \\
\text { acid residues }=1157)\end{array}$ & $\begin{array}{l}32.67 \pm 0.003729 \\
n=4600 \text { frames }\end{array}$ & $\begin{array}{l}43091 \pm 2.9, \mathrm{n}= \\
4600 \text { frames }\end{array}$ \\
\hline 6. & $\begin{array}{l}\text { Fluvastatain- } \\
\text { Helicase complex }\end{array}$ & $\begin{array}{l}3.867 \pm 0.007232 \\
n=4600 \text { frames }\end{array}$ & $\begin{array}{l}1.031 \pm 0.01281,(\text { amino } \\
\text { acid residues }=1157)\end{array}$ & $\begin{array}{l}32.13 \pm 0.003308 \\
n=4600 \text { frames }\end{array}$ & $\begin{array}{l}38259 \pm 2.8, n= \\
4600 \text { frames }\end{array}$ \\
\hline 7. & Free-Spike $_{W T}$ & $\begin{array}{l}8.209 \pm 0.03288 \\
n=4600 \text { frames }\end{array}$ & $\begin{array}{l}3.249 \pm 0.1155 \\
\text { (amino acid residues = } \\
1132 \text { ) }\end{array}$ & $\begin{array}{l}28.82 \pm 0.0213, n \\
=4600\end{array}$ & $\begin{array}{l}43063 \pm 222 \\
n=4600 \text { frames }\end{array}$ \\
\hline 8. & $\begin{array}{l}\text { Fluvastatin-Spike }{ }_{W T} \\
\text { complex }\end{array}$ & $\begin{array}{l}5.745 \pm 0.01281 \\
n=4600 \text { frames }\end{array}$ & $\begin{array}{l}2.035 \pm 0.04672 \\
\text { (amino acid residues }= \\
1132 \text { ) }\end{array}$ & $\begin{array}{l}31.34 \pm 0.005914 \\
\mathrm{n}=4600\end{array}$ & $\begin{array}{l}41807 \pm 234 \\
n=4600 \text { frames }\end{array}$ \\
\hline 9. & Free-Spike $_{D 614 G}$ & $\begin{array}{l}5.865 \pm 0.01874 \\
n=4600 \text { frames }\end{array}$ & $\begin{array}{l}2.747 \pm 0.0595,(\text { amino } \\
\text { acid residues }=1132)\end{array}$ & $\begin{array}{l}31.02 \pm 0.01579 \\
n=4600 \text { frames }\end{array}$ & $\begin{array}{l}43052 \pm 224 \\
n=4600 \text { frames }\end{array}$ \\
\hline 10. & $\begin{array}{l}\text { Fluvastatin- } \\
\text { Spike }_{\text {D614G }} \text { complex }\end{array}$ & $\begin{array}{l}7.334 \pm 0.02686 \\
\mathrm{n}=4600 \text { frames }\end{array}$ & $\begin{array}{l}3.038 \pm 0.06021,(\text { amino } \\
\text { acid residues }=1132)\end{array}$ & $\begin{array}{l}30.34 \pm 0.01316 \\
\mathrm{n}=4600 \text { frames }\end{array}$ & $\begin{array}{l}42901 \pm 229 \\
n=4600 \text { frames }\end{array}$ \\
\hline 11. & $\begin{array}{l}\text { Free } \\
\text { Spike }_{\text {D614G/N501Y }}\end{array}$ & $\begin{array}{l}6.284 \pm 0.02731 \\
n=4600 \text { frames }\end{array}$ & $\begin{array}{l}2.788 \pm 0.02133,(\text { amino } \\
\text { acid residues }=1132)\end{array}$ & $\begin{array}{l}41.71 \pm 0.02195 \\
\mathrm{n}=4600 \text { frames }\end{array}$ & $\begin{array}{l}42885 \pm 3.158 \\
n=4600 \text { frames }\end{array}$ \\
\hline 12. & $\begin{array}{l}\text { Fluvastatin- } \\
\text { Spike }_{\text {D } 614 G / N 501 Y} \\
\text { complex }\end{array}$ & $\begin{array}{l}6.369 \pm 0.02476 \\
n=4600 \text { frames }\end{array}$ & $\begin{array}{l}2.575 \pm 0.02237,(\text { amino } \\
\text { acid residues }=1132)\end{array}$ & $\begin{array}{l}42.58 \pm 0.0165, n \\
=4600 \text { frames }\end{array}$ & $\begin{array}{l}43463 \pm 3.024 \\
n=4600 \text { frames }\end{array}$ \\
\hline
\end{tabular}

\section{RdRp-Fluvastatin complex}

The MD simulation analysis of Fluvastatin-RdRp interaction revealed that the RMSD value of the ligand-protein complex had decreased significantly compared to the free protein (Fig. 7E-7F, Table 3). The free RdRP and fluvastatin-RdRp complex had RMSD values of $3.713 \pm 0.006144$ and $2.584 \pm 0.00357$, respectively (Fig. 7E). This significant reduction in RMSD is in favor of better stability of the fluvastatin-RdRp complex. The RMSD difference was $0.9302 \AA$ (<2 $\AA$ ), suggesting the stability of fluvastatin at the active site of RdRp. Furthermore, the RMSF value was decreased from $0.9124 \pm 0.01 \AA$ (free RdRp) to 0.841 $\pm 0.01 \AA$ due to complexation with fluvastatin. The major regions of fluctuations were highlighted with the color ballot (Fig. 7G-7H). Also, the SASA value of the fluvastatin-RDRP complex $\left(33766 \pm 1.987 \AA^{2}\right)$ was significantly reduced in comparison to free RDRP $\left(38565 \pm 2.269 \AA^{2}\right)$ without any alteration in the radius of gyrations $\left(r_{g}\right)$ values (indicating NO unfolding of the complex) (Fig. S11C-S11D). Thus, the docking of fluvastatin is strong enough so that water can not replace it.

\section{Cl-Pro- Fluvastatin complex}


The MD analysis of fluvastatin-3CL-Pro complex showed the reduction in the average RMSD values of fluvastatin-3CL-Pro complex $(2.576 \pm 0.004 \AA)$ in comparison to the free 3CL-Pro $(2.888 \pm 0.005 \AA)$ (Fig. 7l-7J, Table 3). The conformations of fluvastatin at the beginning and end of MD simulation were superimposed in Fig. 7J, and the difference in RMSD value was $1.78 \AA(<2 \AA)$, suggesting the stability of the fluvastatin-protein complex. The average RMSF value of the amino acid residues was reduced from $0.845 \pm 0.017 \AA$ (free 3 CL-Pro) to $0.7794 \pm 0.016 \AA$ due to complexation with fluvastatin. The major regions of fluctuation were highlighted with a color ballot (Fig. 7K-7L). Moreover, there were no significant changes in the SASA values and $r_{g}$ values (Fig. S11E-S11F, Table 3). These results indicate the formation of a stable complex between fluvastatin and 3 CL-Pro.

\section{$S_{W T^{-}}$Fluvastatin complex, $\mathbf{S}_{\mathrm{D} 614 \mathrm{G}^{-}}$Fluvastatin complex and $\mathbf{S}_{\mathrm{D} 614 \mathrm{G} / \mathrm{N} 501 Y^{-}}$Fluvastatin complex}

As per the RMSD plot, complexation of Spike ${ }_{w t}$ with fluvastatin increased the average RMSD value from $5.95 \pm 1.38 \AA$ (free protein) to $6.25 \pm 1.77 \AA$ (complex) (Fig. 8A, Table 3) The RMSD of superimposed poses of fluvastatin at the beginning and end of the simulation period (Fig. 8B) was $1.0681 \AA(<2 \AA)$. Hence, there were insignificant changes in the backbone of protein and ligand structures, suggesting the stability of the fluvastatin-protein complex.

Further, the average RMSF value after complexation was decreased from $1.03 \pm 0.47 \AA$ (free spike ${ }_{w t}$ protein) to $0.93 \pm 0.422 \AA$ (fluvastatin bound complex). The regions having major changes in RMSF (G1, G2, and G3) were highlighted in Fig. 8C \& 8D. As per the molecular docking study, the fluvastatin binds at the NTD. region (amino acid residue 240 to 250). The RMSF at this region (labeled as $\mathrm{G} 1$ in Fig. $8 \mathrm{C}$ ) was reduced due to Fluvastatin binding, indicating the formation of a stable complex. The SASA plot and the gyration of radius $\left(\mathrm{r}_{\mathrm{g}}\right)$ did not show any significant change, indicating the formation of a stable complex of fluvastatin with spike ${ }_{w t}$ protein (Fig. S12A-S12B).

The comparative RMSD plot of free Spike ${ }_{D 614 G}$ and fluvastatin- Spike ${ }_{D 614 G}$ complex is shown in Fig. 8E. Up to $1100 \mathrm{~ns}$, the free protein and fluvastatin-protein complex had very close RMSD values. After that, the RMSD increased gradually and reached a maximum value of $8.72 \AA$. Thus, there was $1.45 \AA$ ( $<2 \AA)$ enhancement of RMSD at the end of the simulation period. If we consider the poses of fluvastatin at the beginning and end of the simulation period (Fig. 8F), the RMSD value was $1.92 \AA(<2 \AA)$. Again, there was no significant difference in the average RMSD value of free protein $(5.97 \pm 1.13 \AA)$ and fluvastatin-protein bound complex $\left(6.22 \pm 1.68 \AA\right.$ ). Thus, it is evident that fluvastatin- Spike $_{D 614 G}$ is stable, which is further supported by the fact that the average RMSF value after complexation was nearly the same (free protein: $0.98 \pm 0.46 \AA$, fluvastatin bound complex: $0.98 \pm 0.44 \AA$ ). But major reductions in RMSF values of a few amino acid residues (C1, and C2 regions) were observed (Fig. 8G-8H). C2 comprises 800-850 amino acid residues that constitute the fluvastatin binding (S2). No significant changes in The SASA value and gyration of radius $\left(\mathrm{r}_{\mathrm{g}}\right)$ were observed (Fig. S12C-S12D), which suggested forming the stable complex of fluvastatin with the Spike $_{D 614 G}$.

The molecular dynamics studies of fluvastatin- Spike ${ }_{D 614 G / N 501 Y}$ complex showed no significant deviation in the RMSD plot for the free protein or the ligand-protein complex (Fig. 8I-8J). The free protein had an RMSD value of $6.284 \pm 0.027 \AA$, whereas the fluvastatin bound complex had an RMSD value of $6.369 \pm 0.025 \AA$. The curves nearly overlap with each other (Fig. 8I). The RMSD value of poses of fluvastatin at the beginning and end of the simulation period (Fig. 8J) was $1.63 \AA$ (<2 $\AA)$. The average RMSF value of fluvastatin- Spike ${ }_{D 614 G / N 501 Y}$ complex $(2.575 \pm 0.022 \AA)$ was less than that of free protein $(2.788 \pm 0.023 \AA)$, suggesting better stability of the complex. The four regions of Spike ${ }_{D 614 G / N 501 Y}$, having reduced RMSF, were C1: 350-550, C2: 700-780, C3: 820-900, and C4: 980 to 1100, respectively, as highlighted in Fig. 8K-8L. The region C3 constitutes the fluvastatin binding pocket. Hence, the binding of fluvastatin at the docking site of Spike ${ }_{D 614 G / N 501 Y}$ is stable.

Interestingly, The average SASA value was significantly increased from $42885 \pm 3.158 \AA^{2}$ (free protein) to $43463 \pm 3.024 \AA^{2}$ (fluvastatin-protein complex), indicating the opening up loops in the $\mathrm{C} 1$ region (Fig. S12E). Moreover, the free protein and fluvastatin-protein complex had an average radius of gyration $41.71 \pm 0.022 \AA$ and $42.58 \pm 0.017 \AA$, respectively (Fig. S12F). This $2 \%$ increase in the $r_{g}$ also suggested the opening up of loops in the $\mathrm{C} 1$ region. This $\mathrm{C} 1$ region belongs to the RBD

Page $15 / 29$ 
section of the spike protein and is involved in the interaction with the ACE2 receptor. Thus the conformational change in this region of the S-double mutant due to Fluvastatin binding may also interfere with spike-ACE-2 interactions in variant SARSCoV-2 strains.

\section{Discussions}

Since the emergence of the COVID-19 pandemic, there have been many efforts to repurpose the existing drugs, already known to be beneficial to the patients to treat this disease. Several case-control studies showed a positive association between statin usage and reduced mortality of COVID-19 patients hospitalized with this disease $(8,10,58)$. Also, it was hypothesized that since statins can up-regulate ACE2 expression (59), they may prevent coronavirus infection. The direct effect of statins on SARS-CoV-2 was first demonstrated by Gerold et al. (60). They observed that selected statins, especially fluvastatin, significantly reduced the entry of SARS-CoV-2 into the human respiratory cells and genome copy numbers of SARS-CoV-2 in the infected cells. But the mechanism by which statins can inhibit the SARS-Cov-2 progression is not well understood.

As most of the countries are facing the second or third wave of the pandemic, various vaccines have been commercialized for the people to confer protection against SARS-Cov-2. But, the emergence of VOCs acquiring unique mutations in essential viral genes had raised concern about the effectiveness of the vaccines against these novel variants. In a very recent study, it was reported that the neutralizing antibodies from the individuals, those who received one or two doses of either BNT162b2 or mRNA-1273 vaccines, showed limited efficacy against the pseudoviruses representing the globally predominant VOCs (61). This observation was supported by other reports revealing the limited effectiveness of the mRNA vaccines or convalescent plasma against the circulating variants $(62,63)$. Although there are parallel efforts of improving the vaccine efficiency against these variants, there is also the necessity of antiviral medications that can target these variants, harboring the concerned mutations.

In our study, nine statin molecules were screened against four selected target proteins of SARS-CoV2 and the mutated S protein with D614G and N501Y (double mutant) by in silico molecular docking and molecular dynamics study. As per the molecular docking studies, the details of the best-selected candidate drug molecules are tabulated in Table 2. The fluvastatin exhibited a good binding affinity to all the selected target proteins. It binds with the active site of RdRp (remdesivir binding site), 3 CL-Pro (inhibition site), and helicase (ATP binding site). Along with fluvastatin, pitavastatin had shown an equivalent binding affinity for RdRp, and 3-CL-Pro (Table 2). But of all the target proteins, the interaction of fluvastatin with helicase was the best, as a docking score of $-11.3 \mathrm{kcal} / \mathrm{mole}$ was observed. Analysis of the binding site revealed that fluvastatin binds to the ATP-binding site of helicase. Since helicase plays a pivotal role in the replication of the viral genome, binding of fluvastatin might interfere with the activity of the enzyme resulting in inhibition of the viral replication. This finding might justify the inhibitory effect of fluvastatin on SARS-CoV-2 infection, reported by Gerold et al. (30). The Fluvastatin-binding site of helicase consisted of a repetitive unit of proline and alanine (PRO 283, PRO 284, ALA 312, ALA 313, and ALA 316), which contributed to ten hydrophobic interactions with the ligand, resulting in the formation of the most stable complex among all the target proteins. The docking analysis of the interaction of fluvastatin with the modeled wild type S-protein (Spikewt), single mutant S-protein (SpikeD614G), and the double mutant S-protein (SpikeD614G/N501Y) demonstrated an interesting trend. Alteration of the fluvastatin-binding site was observed along with the introduction of the mutation. Interestingly, the total number of interactions between fluvastatin and the mutant S-proteins were also increased compared to the wild-type protein. Overall, the binding affinity of fluvastatin was enhanced with the number of mutations introduced in the S-protein. Previous reports have demonstrated that the introduction of missense mutations like D614G or N501Y had favored an open conformation of the spike-RBD, which ultimately enhanced its affinity for the host receptor $(64,65)$. Not much structural deviation was observed when the mutations D614G or N501Ywere introduced in wild type S-protein (PDB: 7BBH ), and the RMSD values were $<1^{0} \mathrm{~A}$. But these mutations became the determining factors in finding the best docking site, based on the most energetically favorable pose of fluvastatin. During docking, the protein structure remains fixed, but the fluvastatin had rotatable bonds to adjust itself at the docking site. This subtle change in the conformation of S-protein due to the mutations

Page $16 / 29$ 
might have affected the docking pose of the ligand, and hence the binding site of fluvastatin had also altered in the mutant proteins.

Chemical homology, thermodynamic parameters, polarity, and favorable interactions may lead to multiple target sites for a single drug molecule $(66,67)$. Analysis of the binding sites of fluvastatin in all the target proteins (Table S2), revealed that

amino acids like threonine, serine, asparagine, arginine, lysine, and aspartic acid residues were common. Pitavastatin having similar chemical properties like fluvastatin (logP and pKa values) exhibited strong biding affinities to RdRp, 3-CL-Pro, and Sdouble mutant.

\section{Conclusion}

Although the major limitation of this work is that it is based on a computational prediction without any laboratory validation, but such studies are significant for the research groups performing wet-lab experiments intending to identify novel antiviral compounds. The most encouraging result obtained from our study is that fluvastatin emerged as the best drug candidate from the blind docking studies with all the nine statin molecules (Fig. 9), which corroborates with the recently published functional study (30). Thus our research will help to predict the molecular mechanism by which this drug inhibits SARS-CoV2 pathogenesis.

\section{Declarations}

\section{Funding and Acknowledgement}

We did not receive any funding or grants from any funding agency for this work. We acknowledge NIBMG for the administrative support. We are also thankful to Prof. Partha P. Majumder, Distinguished Professor, NIBMG, for the encouragement and support.

\section{Author contribution}

DG, DGD, GC and AD designed the study, performed analysis, and wrote the manuscript. KR, AG, NS, and NKB analyzed data and helped in manuscript writing. All authors have read and approved the final manuscript.

\section{Declaration of competing interest}

The authors have no conflict of interest to declare.

\section{Ethics approval and consent to participate}

NA (The study does not contain any human participants, human data or human tissue experiment)

\section{Consent for publication}

NA (The study does not contain any individual person's data in any form)

\section{Availability of Data and Materials}

NA

\section{References}

1. Bravi F, Flacco ME, Carradori T, Volta CA, Cosenza G, De Togni A, et al. Predictors of severe or lethal COVID-19, including Angiotensin Converting Enzyme inhibitors and Angiotensin II Receptor Blockers, in a sample of infected Italian citizens. PLoS One. 2020;15(6):e0235248. 
2. Chan JF, Yuan S, Kok KH, To KK, Chu H, Yang J, et al. A familial cluster of pneumonia associated with the 2019 novel coronavirus indicating person-to-person transmission: a study of a family cluster. Lancet (London, England). 2020;395(10223):514-23.

3. Orgnanization WH. WHO Coronavirus (COVID-19) Dashboard 2021 [cited 2021 18.05.2021]. Available from: https://covid19.who.int/.

4. Narayanan K, Maeda A, Maeda J, Makino S. Characterization of the coronavirus M protein and nucleocapsid interaction in infected cells. J Virol. 2000;74(17):8127-34.

5. Zhu N, Zhang D, Wang W, Li X, Yang B, Song J, et al. A Novel Coronavirus from Patients with Pneumonia in China, 2019. The New England journal of medicine. 2020;382(8):727-33.

6. McKee DL, Sternberg A, Stange U, Laufer S, Naujokat C. Candidate drugs against SARS-CoV-2 and COVID-19. Pharmacol Res. 2020;157:104859-.

7. Biswas N, Majumder P. Analysis of RNA sequences of 3636 SARS-CoV-2 collected from 55 countries reveals selective sweep of one virus type. 2020;151(5):450-8.

8. Korber B, Fischer WM, Gnanakaran S, Yoon H, Theiler J, Abfalterer W, et al. Tracking Changes in SARS-CoV-2 Spike: Evidence that D614G Increases Infectivity of the COVID-19 Virus. Cell. 2020;182(4):812-27.e19.

9. Bhattacharyya C, Das C, Ghosh A, Singh AK, Mukherjee S, Majumder PP, et al. SARS-CoV-2 mutation 614G creates an elastase cleavage site enhancing its spread in high AAT-deficient regions. Infection, Genetics and Evolution. 2021;90:104760.

10. Burki T. Understanding variants of SARS-CoV-2. Lancet (London, England). 2021;397(10273):462.

11. SARS-CoV-2 Variants: WHO; 2020 [Available from: https://www.who.int/csr/don/31-december-2020-sars-cov2variants/en/.

12. Luan B, Wang H, Huynh T. Molecular Mechanism of the N501Y Mutation for Enhanced Binding between SARS-CoV-2's Spike Protein and Human ACE2 Receptor. bioRxiv. 2021:2021.01.04.425316.

13. Castro A, Carter H, Zanetti M. Potential global impact of the N501Y mutation on MHC-II presentation and immune escape2021.

14. Gupta RK. Will SARS-CoV-2 variants of concern affect the promise of vaccines? Nature Reviews Immunology. 2021.

15. Horby P, Mafham M, Linsell L, Bell JL, Staplin N, Emberson JR, et al. Effect of Hydroxychloroquine in Hospitalized Patients with COVID-19: Preliminary results from a multi-centre, randomized, controlled trial. medRxiv. 2020:2020.07.15.20151852.

16. Beigel JH, Tomashek KM, Dodd LE, Mehta AK, Zingman BS, Kalil AC, et al. Remdesivir for the Treatment of Covid-19 Final Report. The New England journal of medicine. 2020;383(19):1813-26.

17. Rochwerg B, Siemieniuk RAC, Agoritsas T, Lamontagne F, Askie L, Lytvyn L, et al. A living WHO guideline on drugs for covid-19. BMJ. 2020;370:m3379.

18. Zeiser R. Immune modulatory effects of statins. Immunology. 2018;154(1):69-75.

19. Minz MM, Bansal M, Kasliwal RR. Statins and SARS-CoV-2 disease: Current concepts and possible benefits. Diabetes Metab Syndr. 2020;14(6):2063-7.

20. Castiglione V, Chiriacò M, Emdin M, Taddei S, Vergaro G. Statin therapy in COVID-19 infection. Eur Heart J Cardiovasc Pharmacother. 2020;6(4):258-9.

21. Verpaalen B, Neyts J, Delang L. Are statins a viable option for the treatment of infections with the hepatitis $C$ virus? Antiviral Research. 2014;105:92-9.

22. Españo E, Nam J-H, Song E-J, Song D, Lee C-K, Kim J-K. Lipophilic statins inhibit Zika virus production in Vero cells. Scientific Reports. 2019;9(1):11461.

23. Gorabi AM, Kiaie N, Bianconi V, Jamialahmadi T, Al-Rasadi K, Johnston TP, et al. Antiviral effects of statins. Progress in Lipid Research. 2020;79:101054. 
24. Fedson DS. Treating influenza with statins and other immunomodulatory agents. Antiviral Res. 2013;99(3):417-35.

25. Vandermeer ML, Thomas AR, Kamimoto L, Reingold A, Gershman K, Meek J, et al. Association between use of statins and mortality among patients hospitalized with laboratory-confirmed influenza virus infections: a multistate study. J Infect Dis. 2012;205(1):13-9.

26. Gupta A, Madhavan MV, Poterucha TJ, DeFilippis EM, Hennessey JA, Redfors B, et al. Association Between Antecedent Statin Use and Decreased Mortality in Hospitalized Patients with COVID-19. Res Sq. 2020:rs.3.rs-56210.

27. Zhang XJ, Qin JJ, Cheng X, Shen L, Zhao YC, Yuan Y, et al. In-Hospital Use of Statins Is Associated with a Reduced Risk of Mortality among Individuals with COVID-19. Cell Metab. 2020;32(2):176-87.e4.

28. Daniels LB, Sitapati AM, Zhang J, Zou J, Bui QM, Ren J, et al. Relation of Statin Use Prior to Admission to Severity and Recovery Among COVID-19 Inpatients. Am J Cardiol. 2020;136:149-55.

29. De Spiegeleer A, Bronselaer A, Teo JT, Byttebier G, De Tré G, Belmans L, et al. The Effects of ARBs, ACEis, and Statins on Clinical Outcomes of COVID-19 Infection Among Nursing Home Residents. J Am Med Dir Assoc. 2020;21(7):909-14.e2.

30. Zapatero-Belinchón FJ, Moeller R, Lasswitz L, van Ham M, Becker M, Brogden G, et al. Fluvastatin mitigates SARS-CoV-2 infection in human lung cells. medRxiv. 2021:2020.07.13.20152272.

31. Waterhouse A, Bertoni M, Bienert S, Studer G, Tauriello G, Gumienny R, et al. SWISS-MODEL: homology modelling of protein structures and complexes. Nucleic Acids Res. 2018;46(W1):W296-w303.

32. Krieger E, Joo K, Lee J, Lee J, Raman S, Thompson J, et al. Improving physical realism, stereochemistry, and side-chain accuracy in homology modeling: Four approaches that performed well in CASP8. Proteins: Structure, Function, and Bioinformatics. 2009;77(S9):114-22.

33. Waszkowycz B. Towards improving compound selection in structure-based virtual screening. Drug Discovery Today. 2008;13(5):219-26.

34. Harder E, Damm W, Maple J, Wu C, Reboul M, Xiang JY, et al. OPLS3: A Force Field Providing Broad Coverage of Druglike Small Molecules and Proteins. Journal of Chemical Theory and Computation. 2016;12(1):281-96.

35. Ban T, Ohue M, Akiyama Y. Multiple grid arrangement improves ligand docking with unknown binding sites: Application to the inverse docking problem. Computational Biology and Chemistry. 2018;73:139-46.

36. Dhasmana A, Raza S, Jahan R, Lohani M, Arif JM. Chapter 19 - High-Throughput Virtual Screening (HTVS) of Natural Compounds and Exploration of Their Biomolecular Mechanisms: An In Silico Approach. In: Ahmad Khan MS, Ahmad I, Chattopadhyay D, editors. New Look to Phytomedicine: Academic Press; 2019. p. 523-48.

37. Discovery Studio Visualization [Internet] [updated 2016. Available from: http://accelrys.com/products/collaborativescience/biovia-discovery-studio/visualization-download.php.

38. Humphrey W, Dalke A, Schulten K. VMD: visual molecular dynamics. Journal of molecular graphics. 1996;14(1):33-8, 278.

39. Hsin J, Arkhipov A, Yin Y, Stone JE, Schulten K. Using VMD: an introductory tutorial. Curr Protoc Bioinformatics. 2008;Chapter 5:Unit-5.7.

40. Phillips JC, Hardy DJ, Maia JDC, Stone JE, Ribeiro JV, Bernardi RC, et al. Scalable molecular dynamics on CPU and GPU architectures with NAMD. The Journal of chemical physics. 2020;153(4):044130.

41. Aldeghi M, Heifetz A, Bodkin MJ, Knapp S, Biggin PC. Predictions of Ligand Selectivity from Absolute Binding Free Energy Calculations. Journal of the American Chemical Society. 2017;139(2):946-57.

42. Onufriev AV, Case DA. Generalized Born Implicit Solvent Models for Biomolecules. Annu Rev Biophys. 2019;48:275-96.

43. Paquet E, Viktor HL. Molecular Dynamics, Monte Carlo Simulations, and Langevin Dynamics: A Computational Review. BioMed Research International. 2015;2015:183918.

44. Pastor RW, Brooks BR, Szabo A. An analysis of the accuracy of Langevin and molecular dynamics algorithms. Molecular Physics. 1988;65(6):1409-19. 
45. Pedretti A, Villa L, Vistoli G. VEGA: A versatile program to convert, handle and visualize molecular structure on Windowsbased PCs. Journal of molecular graphics \& modelling. 2002;21:47-9.

46. Zhou B, Thao TTN, Hoffmann D, Taddeo A, Ebert N, Labroussaa F, et al. SARS-CoV-2 spike D614G change enhances replication and transmission. Nature. 2021;592(7852):122-7.

47. Ozono S, Zhang Y, Ode H, Sano K, Tan TS, Imai K, et al. SARS-CoV-2 D614G spike mutation increases entry efficiency with enhanced ACE2-binding affinity. Nature Communications. 2021;12(1):848.

48. Ali F, Kasry A, Amin M. The new SARS-CoV-2 strain shows a stronger binding affinity to ACE2 due to N501Y mutant. Medicine in Drug Discovery. 2021;10:100086.

49. Ali F, Kasry A, Amin M. The new SARS-CoV-2 strain shows a stronger binding affinity to ACE2 due to N501Y mutant. Med Drug Discov. 2021;10:100086.

50. Yin W, Mao C, Luan X, Shen D-D, Shen Q, Su H, et al. Structural basis for inhibition of the RNA-dependent RNA polymerase from SARS-CoV-2 by remdesivir. Science. 2020;368(6498):1499-504.

51. Jin Z, Du X, Xu Y, Deng Y, Liu M, Zhao Y, et al. Structure of Mpro from SARS-CoV-2 and discovery of its inhibitors. Nature. 2020;582(7811):289-93.

52. Reiner Ž, Hatamipour M, Banach M, Pirro M, Al-Rasadi K, Jamialahmadi T, et al. Statins and the COVID-19 main protease: in silico evidence on direct interaction. Arch Med Sci. 2020;16(3):490-6.

53. Ivanov KA, Ziebuhr J. Human coronavirus 229E nonstructural protein 13: characterization of duplex-unwinding, nucleoside triphosphatase, and RNA 5'-triphosphatase activities. J Virol. 2004;78(14):7833-8.

54. Mirza MU, Froeyen M. Structural elucidation of SARS-CoV-2 vital proteins: Computational methods reveal potential drug candidates against main protease, Nsp12 polymerase and Nsp13 helicase. Journal of Pharmaceutical Analysis. 2020.

55. White MA, Lin W, Cheng X. Discovery of COVID-19 Inhibitors Targeting the SARS-CoV-2 Nsp13 Helicase. The Journal of Physical Chemistry Letters. 2020;11(21):9144-51.

56. Wang Q, Zhang Y, Wu L, Niu S, Song C, Zhang Z, et al. Structural and Functional Basis of SARS-CoV-2 Entry by Using Human ACE2. Cell. 2020;181(4):894-904.e9.

57. Cai Y, Zhang J, Xiao T, Peng H, Sterling SM, Walsh RM, et al. Distinct conformational states of SARS-CoV-2 spike protein. Science. 2020;369(6511):1586-92.

58. Gupta S, Hayek SS, Wang W, Chan L, Mathews KS, Melamed ML, et al. Factors Associated With Death in Critically III Patients With Coronavirus Disease 2019 in the US. JAMA internal medicine. 2020;180(11):1-12.

59. Li Y-H, Wang Q-X, Zhou J-W, Chu X-M, Man Y-L, Liu P, et al. Effects of rosuvastatin on expression of angiotensinconverting enzyme 2 after vascular balloon injury in rats. J Geriatr Cardiol. 2013;10(2):151-8.

60. Moeller R, Zapatero-Belinchón FJ, Lasswitz L, Kirui J, Brogden G, Gunesch AP, et al. Effect of statins on SARS-CoV-2 infection. medRxiv. 2020:2020.07.13.20152272.

61. Garcia-Beltran WF, Lam EC, St. Denis K, Nitido AD, Garcia ZH, Hauser BM, et al. Multiple SARS-CoV-2 variants escape neutralization by vaccine-induced humoral immunity. Cell. 2021;184(9):2372-83.e9.

62. Wang Z, Schmidt F, Weisblum Y, Muecksch F, Barnes CO, Finkin S, et al. mRNA vaccine-elicited antibodies to SARS-CoV-2 and circulating variants. Nature. 2021;592(7855):616-22.

63. Wang P, Nair MS, Liu L, Iketani S, Luo Y, Guo Y, et al. Antibody resistance of SARS-CoV-2 variants B.1.351 and B.1.1.7. Nature. 2021;593(7857):130-5.

64. Mansbach RA, Chakraborty S, Nguyen K, Montefiori DC, Korber B, Gnanakaran S. The SARS-CoV-2 Spike variant D614G favors an open conformational state. Science Advances. 2021;7(16):eabf3671.

65. Jakhmola S, Indari O, Kashyap D, Varshney N, Das A, Manivannan E, et al. Mutational analysis of structural proteins of SARS-CoV-2. Heliyon. 2021;7(3):e06572.

66. Zhang W, Pei J, Lai L. Computational Multitarget Drug Design. Journal of Chemical Information and Modeling. 2017;57(3):403-12. 
67. Murugan NA, Kumar S, Jeyakanthan J, Srivastava V. Searching for target-specific and multi-targeting organics for Covid19 in the Drugbank database with a double scoring approach. Scientific Reports. 2020;10(1):19125.

\section{Figures}
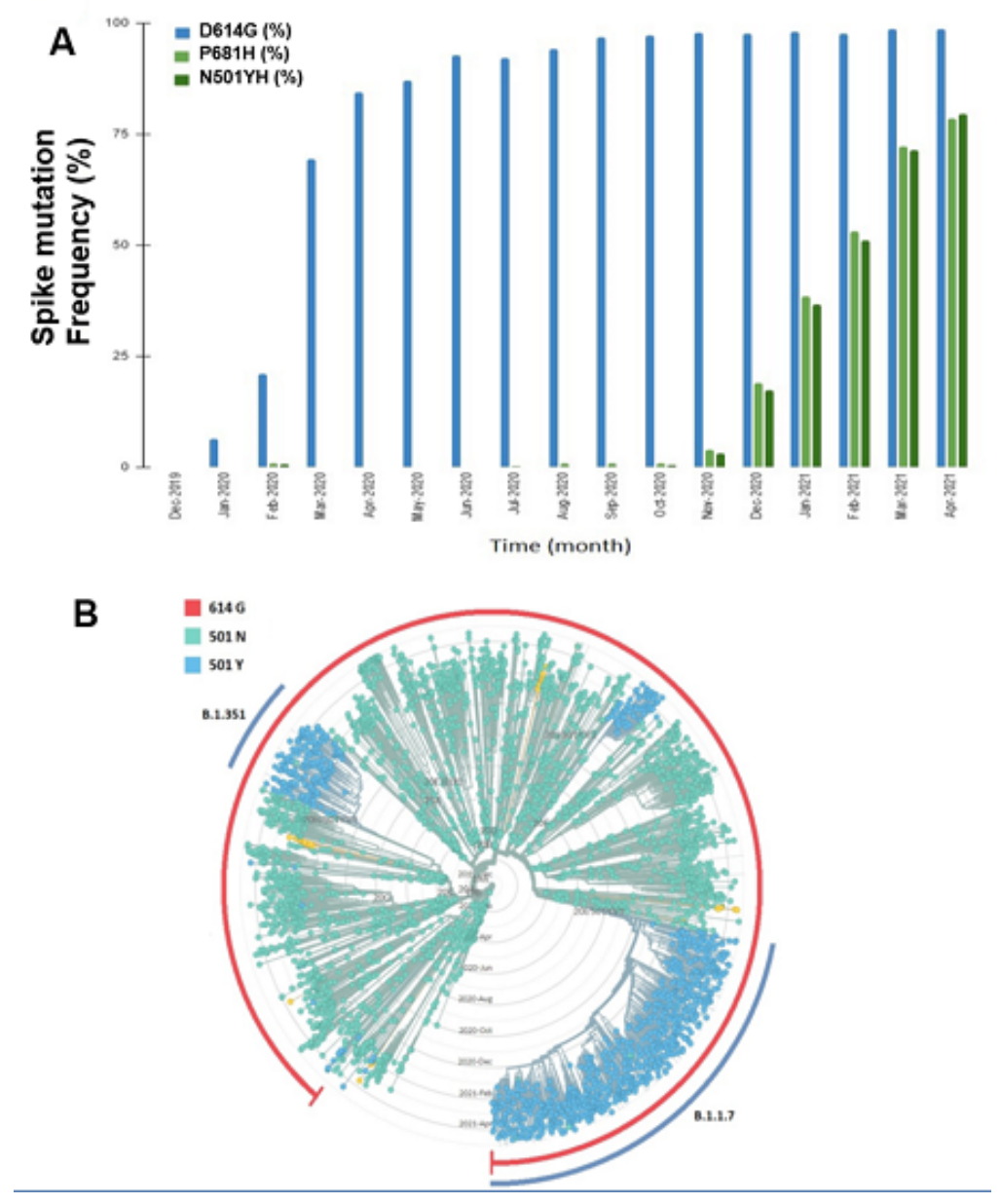

\section{Figure 1}

(A) Temporal frequency of frequently mutated SARS-CoV-2 spike protein variants, D614G, P681H, and N501Y. The D614G mutation became predominant worldwide in March 2020. The N501Y andP681H mutations started gaining high prevalence from December 2020 and became predominant three months down the line. (B) Phylodynamic timetree analysis using the global subset of SARS-CoV-2sequences. Each concentric circles represent the time of sequence collection with origin at the center. Eachsmall circle represents a sequence. The SARS-CoV-2 sequences that carry D614G mutations aremarked in a redcolored semi-circle. 


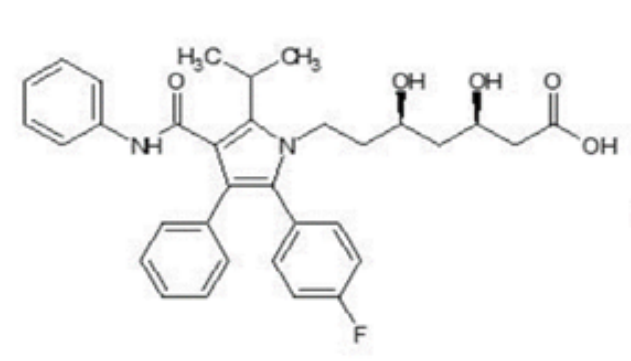

Atorvastatin

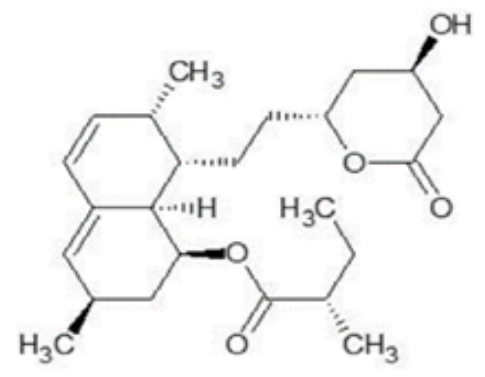

Lovastatin

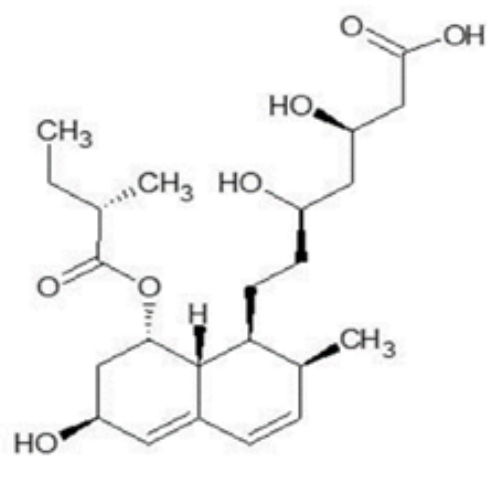

Pravastatin<smiles>COCc1c(C(C)C)nc(C(C)C)c(/C=C/C(O)C[C@H](O)CC(=O)O)c1-c1ccc(F)cc1</smiles>

Cerivastatin<smiles>CCC(C)C(=O)OC1CCC=C2C=CC(C)C(CCC3CC(O)CC(=O)O3)[C@]21O</smiles>

Mevastatin

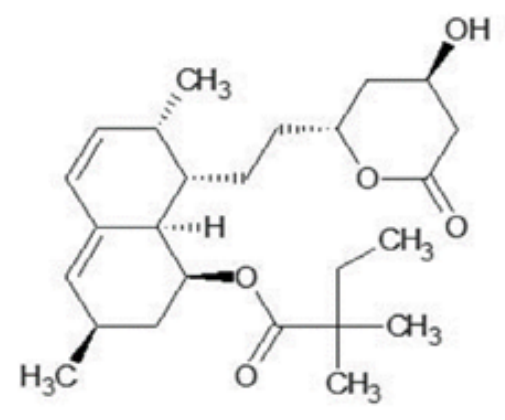

Simvastatin

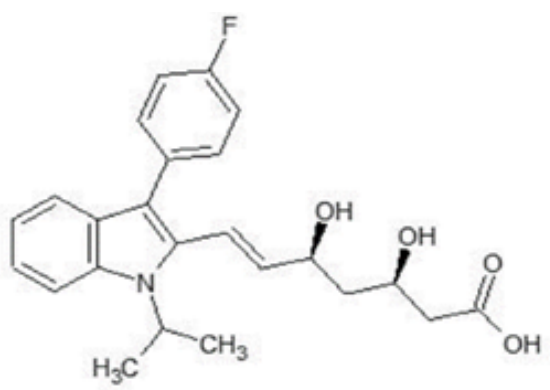

Fluvastatin

\section{Pitavastatin}<smiles>CC(C)c1nc(N(C)S(C)(=O)=O)nc(-c2ccc(F)cc2)c1/C=C/C(O)CC(O)CC(=O)O</smiles>

$\underline{\text { Rosuvastatin }}$

Figure 2

2D structure of the statin molecules. 

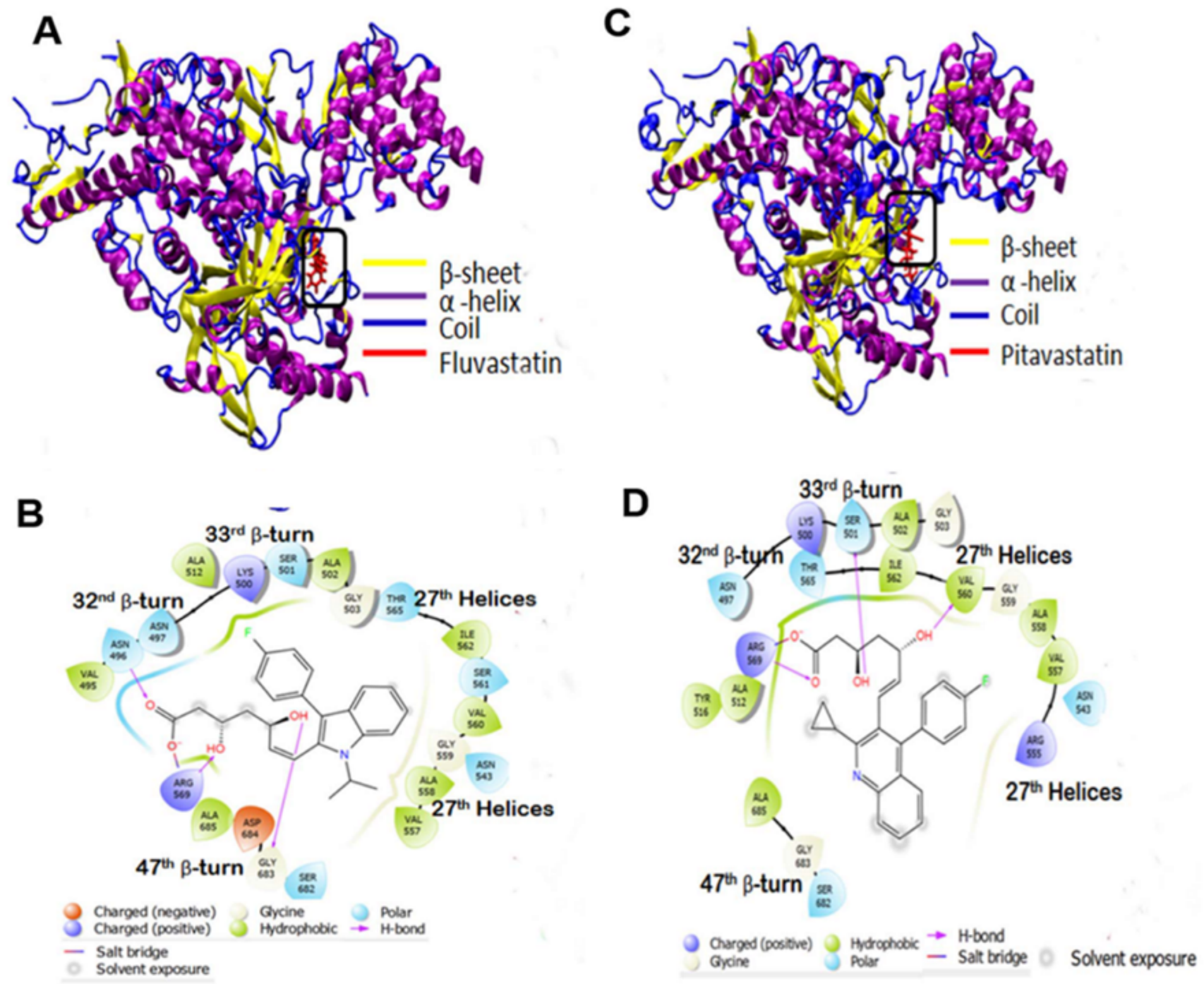

Figure 3

Molecular docking study of fluvastatin and pitavastatin to RdRp. A,C: 3D diagram of fluvastatin-RdRp complex and pitavastatin-RdRp complex, respectively. B,D: 2D diagram for representation of interactions of ligand molecule with the amino acid residues at the binding site; B:Fluvastatin-RdRp complex and D: Pitavastatin-RdRp complex. 

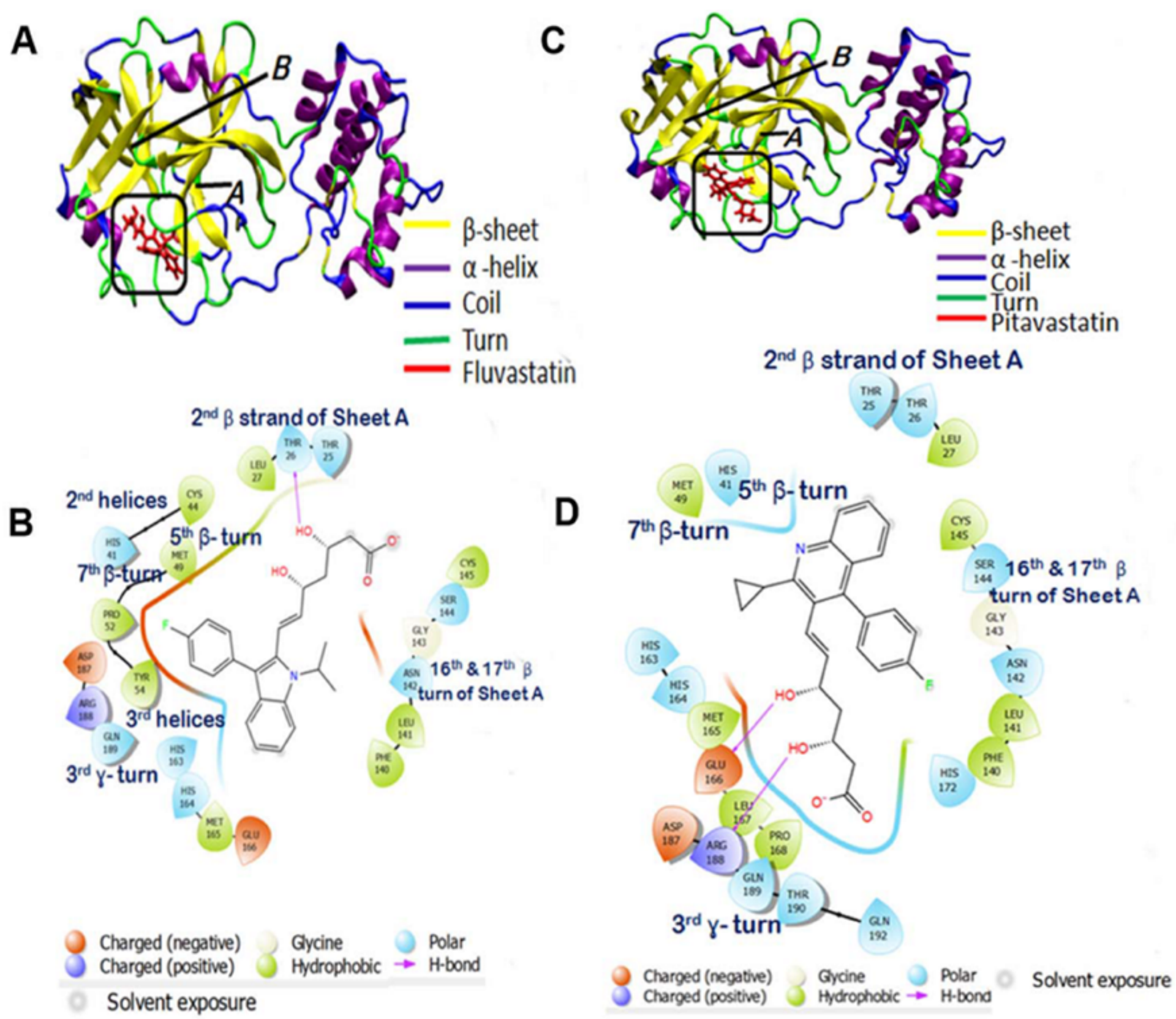

Figure 4

Molecular docking study of fluvastatin and pitavastatin to 3-Cl-Pro. A,C: 3D diagram of fluvastatin-3-Cl-Pro complex and pitavastatin-3-Cl-Pro complex, respectively. B,D: 2D diagram for representation of interactions of ligand molecule with the amino acid residues at the binding site; B:Fluvastatin-RdRp complex and D: Pitavastatin-RdRp complex 


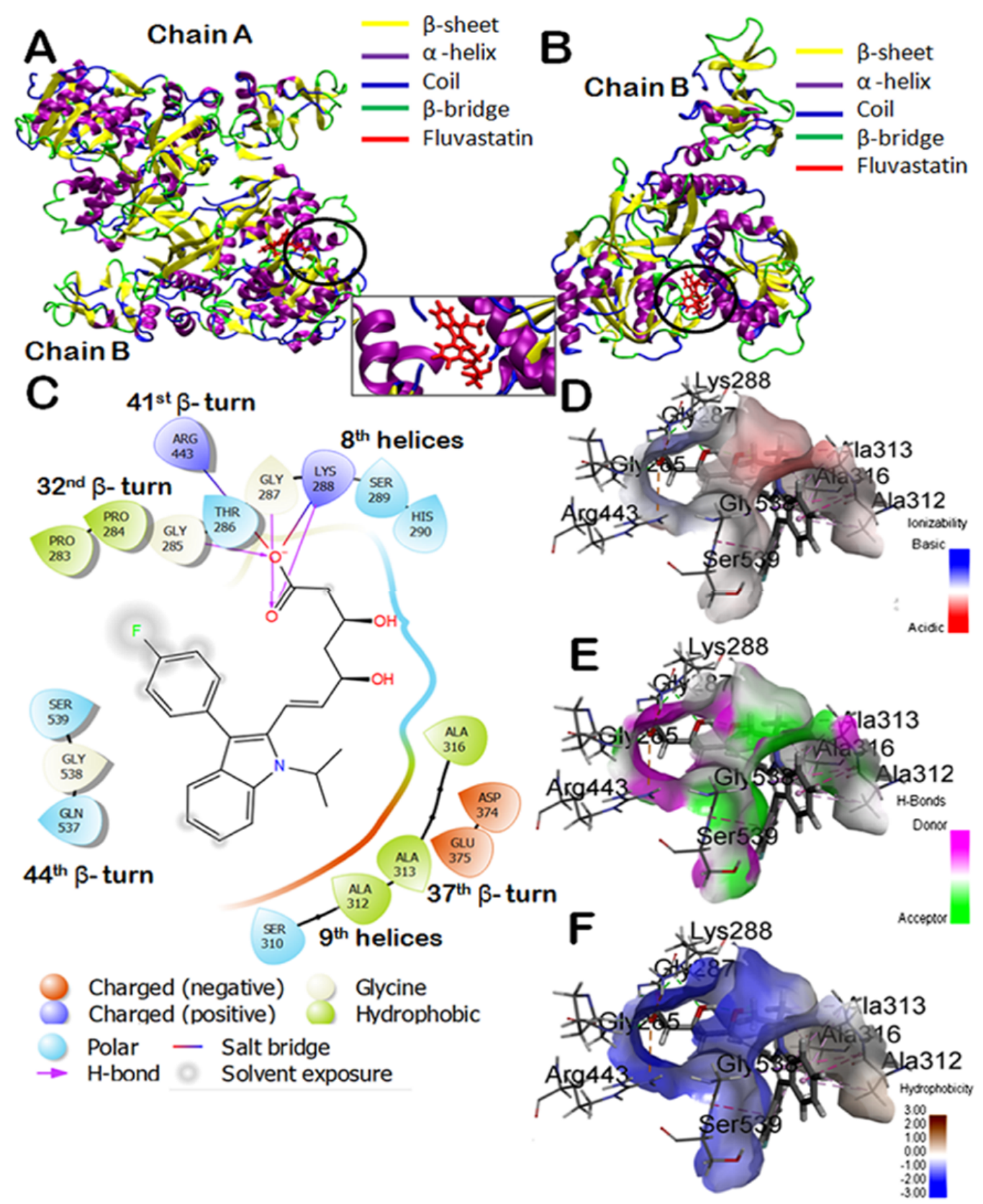

Figure 5

Molecular docking study of fluvastatin to helicase. A: 3D diagram of ligand-protein complex (chain A and Chain B). B: 3D diagram of fluvastatin bound helicase complex (chain B only). C: 2D diagram for representation of interactions of fluvastatin with the amino acid residues at the binding site. D-F: Mapping of binding site cavity according to ionizability, H-bond donoracceptor residue, and hydrophobicity, respectively. 

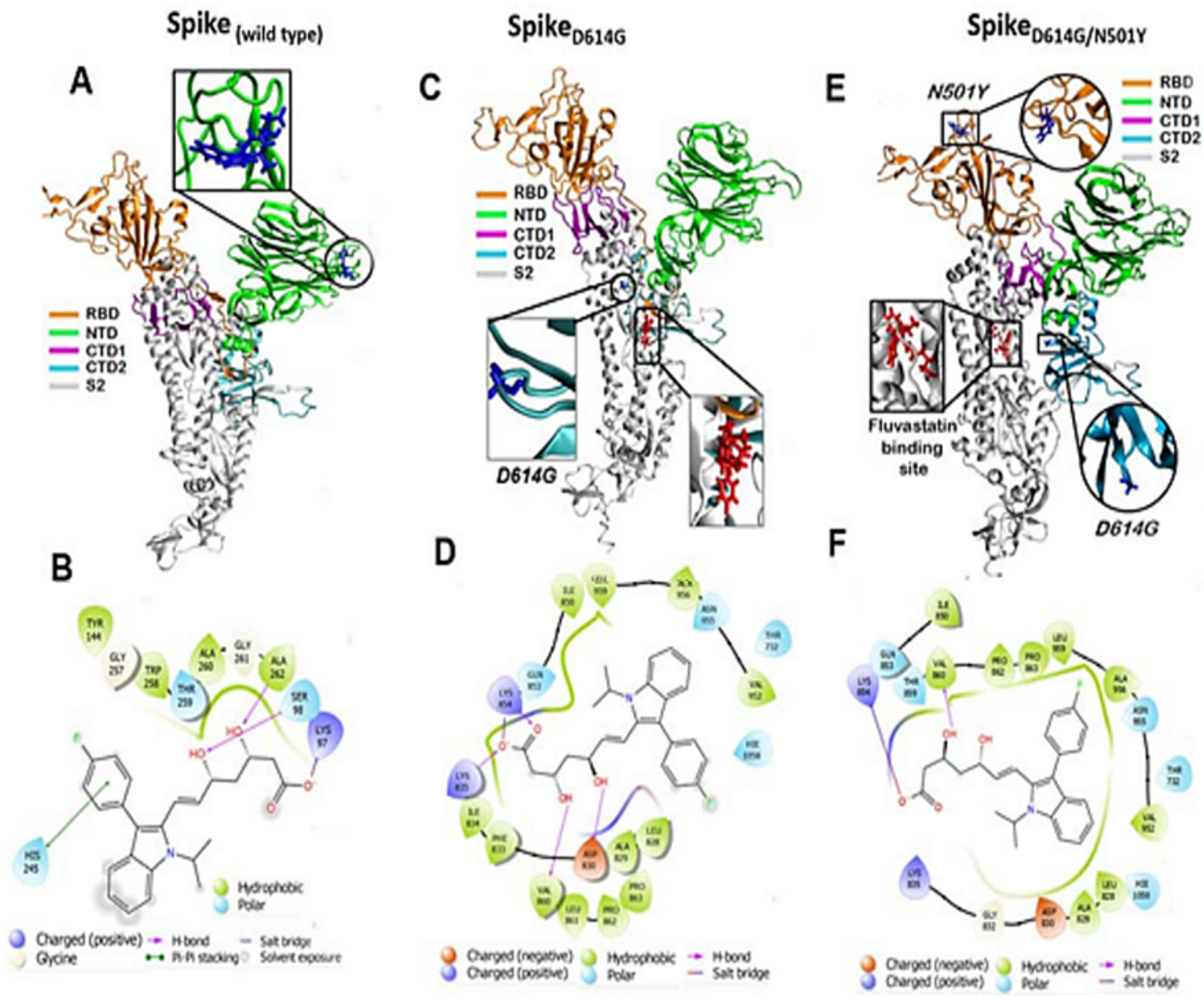

Figure 6

Molecular docking study of fluvastatin to SpikeWT, SpikeD614G, SpikeD614G/N501Y. A,C,E: 3D diagram of fluvastatinSpikeWT complex, fluvastatin-SpikeD614G complex, and fluvastatin- SpikeD614G/N501Y complex respectively. B,D,F: 2D diagram for representation of interactions of fluvastatin molecule with the amino acid residues at the binding site of SpikeWT, SpikeD614G, and SpikeD614G/N501Y,respectively. 

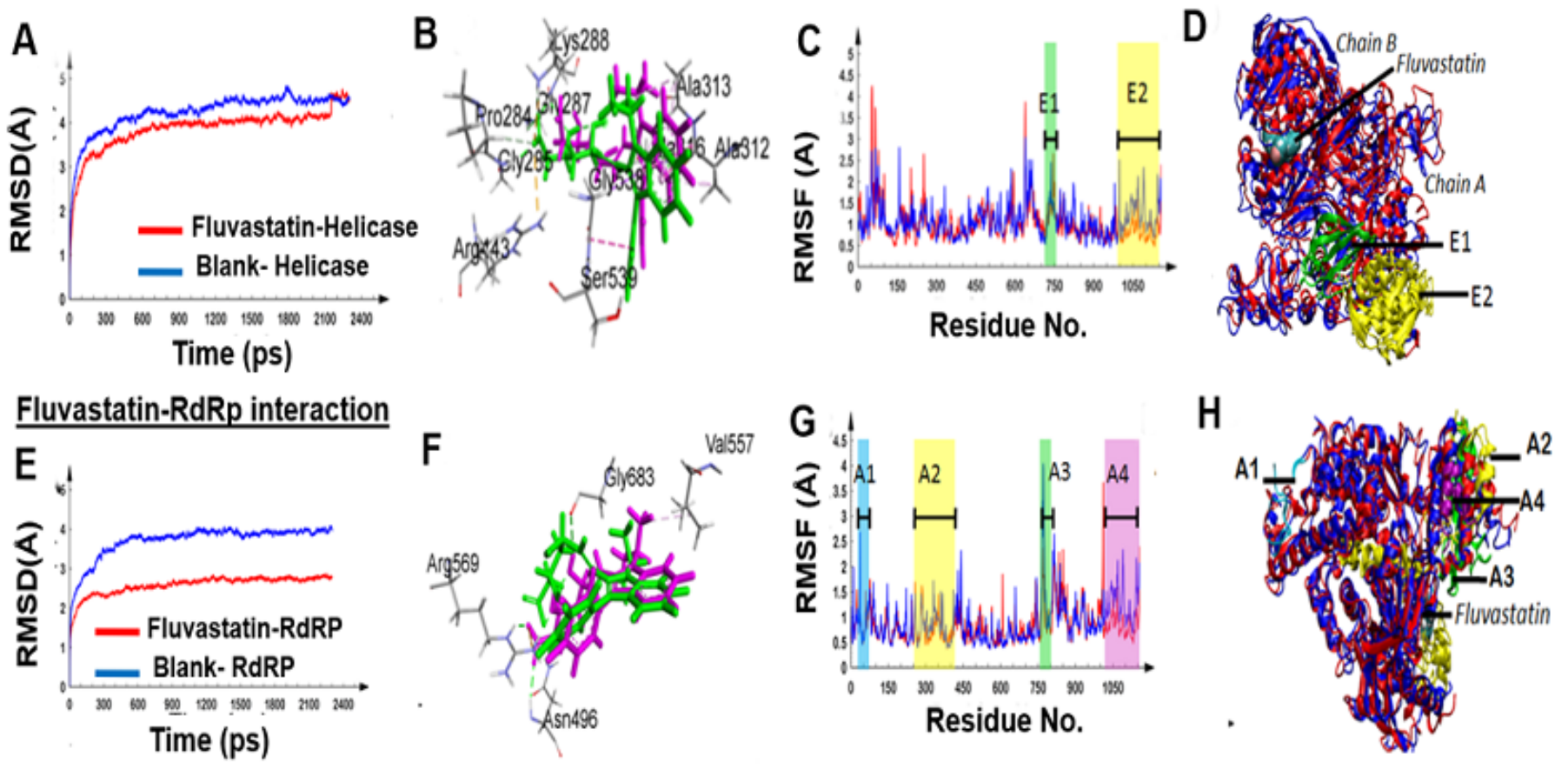

Fluvastatin-3CL-Pro interaction
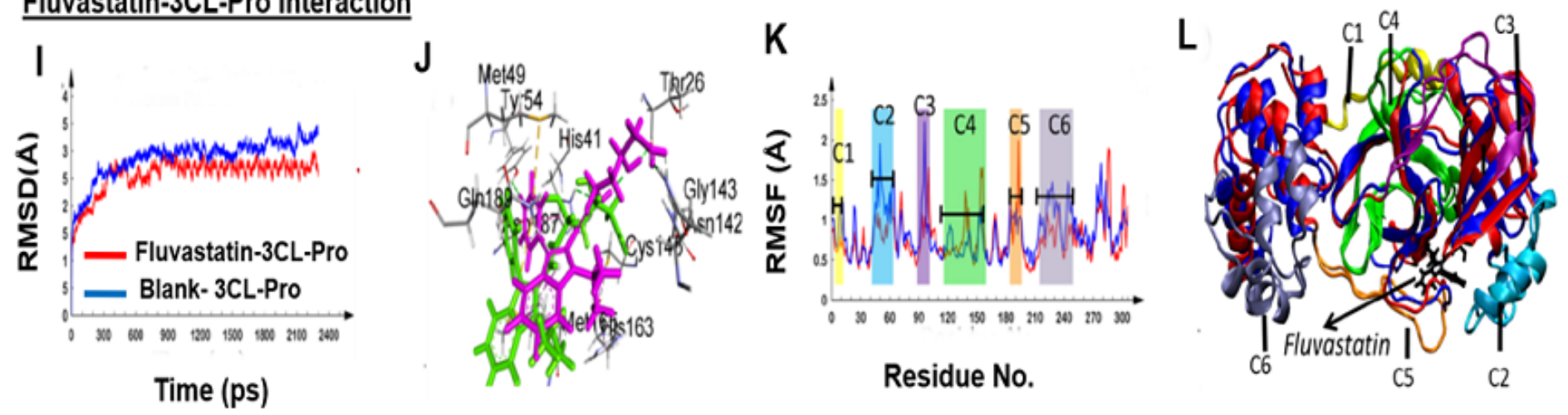

Figure 7

Molecular dynamics simulation studies of Fluvastatin with Helicase, RdRp, and 3-Cl-Pro. A-D: fluvastatin-helicase complex, E$\mathrm{H}$ : fluvastatin-RdRp complex, and I-L: fluvastatin-3Cl-Pro complex. A,E,I: RMSD plot of blank protein (blue color) and fluvastatin-protein complexes (red color). B,F,J: Superimposed poses of fluvastatin at the beginning (purple color) and end (green) of the simulation period. C,G,K: Comparative representation of RMSF plot of amino acid residues of the free protein molecule (blue color) and protein-ligand complex (red color). Significant fluctuations are highlighted as the different colored ballots. D,H,L: Superimposed structure of free protein and fluvastatin bound complex at the end of the simulation period. Regions with major fluctuations have been mapped on the 3D structures. 

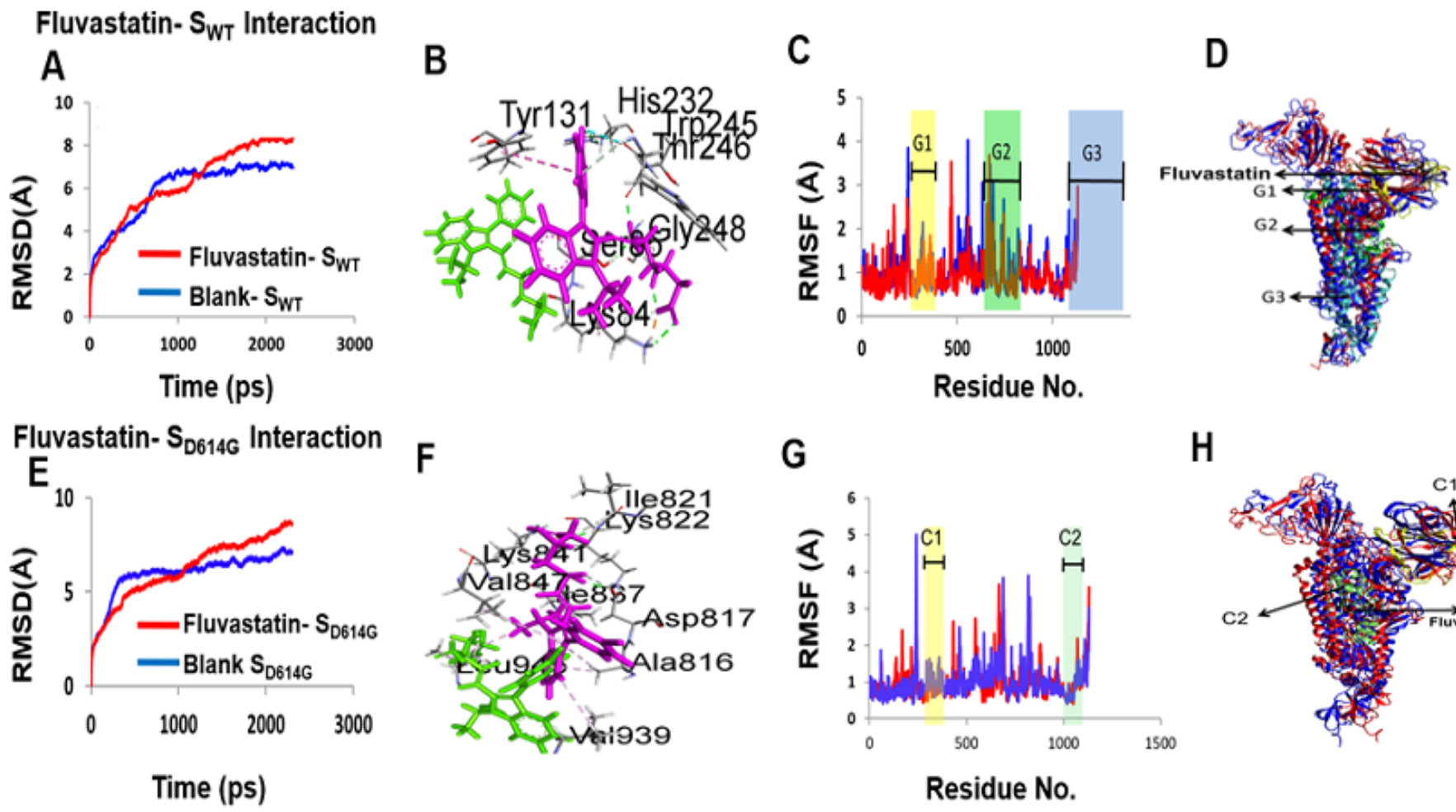

G
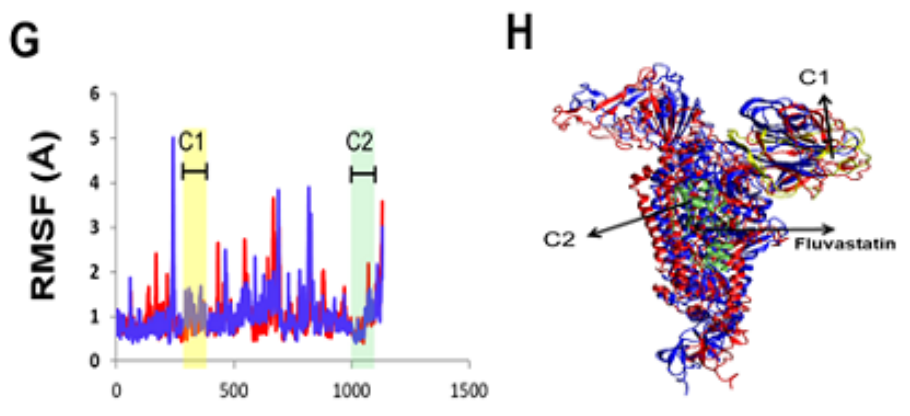

Residue No.
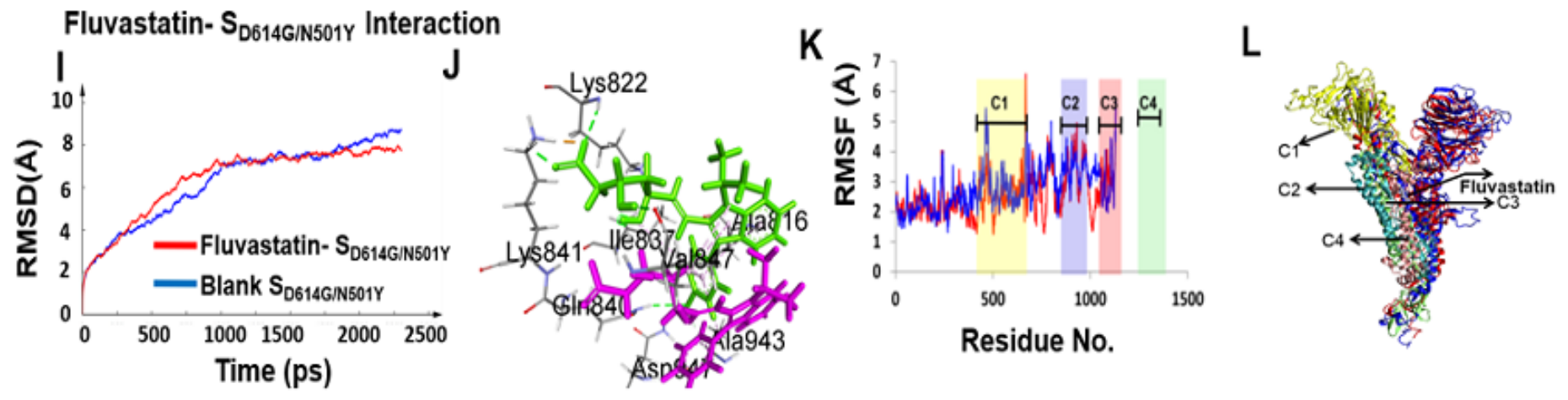

Figure 8

Molecular dynamics simulation studies of fluvastatin with spike wild type and mutant proteins. A-D: fluvastatin-SpikeWT complex, E-H: fluvastatin-SpikeD614G complex, and I-L: fluvastatin-SpikeD614G/N501Y complex. A,E,I: RMSD plot of blank protein (blue color) and fluvastatin-protein complexes (red color). B,F,J: Superimposed poses of fluvastatin at the beginning (purple color) and end (green) of the simulation period. C,G,K: Comparative representation of RMSF plot of amino acid residues of the free protein molecule (blue color) and protein-ligand complex (red color). Major fluctuations are highlighted as the different colored ballots. D,H,L: Superimposed structure of free protein and fluvastatin bound complex at the end of the simulation period. Regions with major fluctuations have been mapped into the 3D structures. 


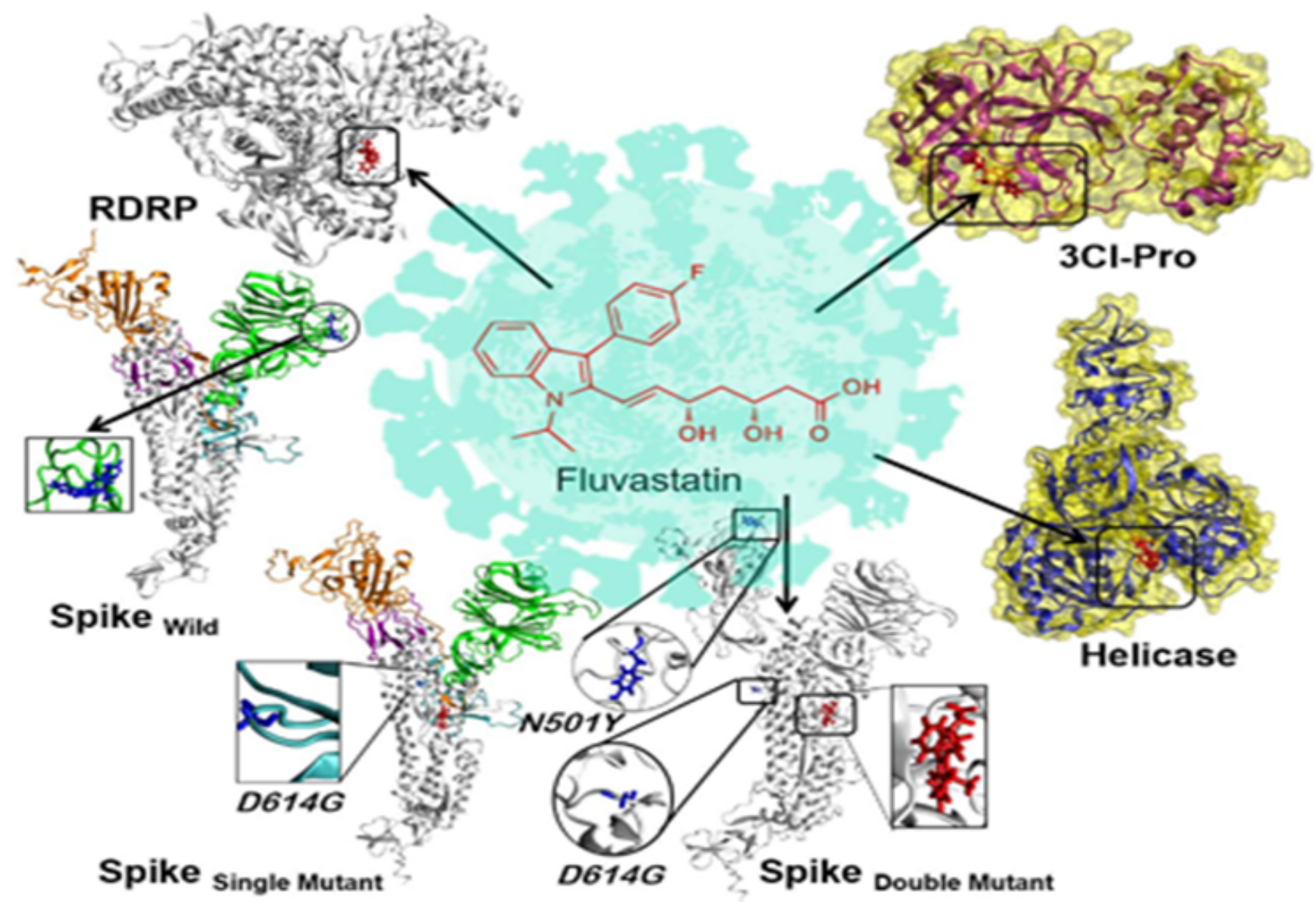

Figure 9

Illustration to define the possible mechanism of fluvastatin against SARS-CoV-2

\section{Supplementary Files}

This is a list of supplementary files associated with this preprint. Click to download.

- SupplementaryMaterials.docx 